\title{
Local tuning and power requirements of a multi-input multi-output decentralised velocity feedback with inertial actuators
}

\author{
Stefano Camperi*, Maryam Ghandchi-Tehrani, Stephen J. Elliott \\ Institute of Sound and Vibration Research, Highfield, Southampton SO171BJ, UK
}

\begin{abstract}
This paper discusses the tuning of multiple decentralised velocity feedback units for broadband vibration control on a plate using inertial actuators. An analysis of the performance is presented in terms of maximising the mechanical power absorbed and of the control effort, defined by the electrical power in the control units, and the results are validated numerically. Two inertial actuators are mounted on an experimental plate, and driven with control currents proportional to the measured local velocities of the plate, collocated with the actuators. Tuning is compared by using either the measurement of kinetic energy of the plate, or the mechanical power absorbed by the control units from the plate. It is found that the mechanical power absorbed can be used as a local cost function to tune the control units, as an alternative to the kinetic energy, not only when reactive actuators are used, but also when inertial actuators are in use. In this way, the tuning process is greatly simplified, since the mechanical power absorbed can be measured directly from the feedback velocity signal and a knowledge of the actuators dynamics, without further information on the plate dynamics. The optimal gain for each unit is found to be reasonably independent of the gain implemented on the other unit. This implies that the system is robust against failure of a unit, since the other would still be optimally tuned. However, this tuning process depends on many factors, such as the type, position and number of control units implemented, and a comparison between different active control solutions, with respect to the optimal feedback gains only is not practical. For this reason, the measure of the electrical power, required by the control system, is suggested as a direct estimation of the control effort. To give an example of the proposed approach, the performance of the two control units is compared with the case in which only one unit at the time is active. The two-channel architecture is found not only to outperform the single-channel one in terms of vibration reduction, but also in terms of consumed electrical power. When the two channels are active, more than $8 \mathrm{~dB}$ broadband reduction is measured, and the consumed electrical power is found to be similar to the one consumed when only one unit is operating, which is optimally tuned.
\end{abstract}

Keywords:

local tuning, decentralised, power, inertial actuator, velocity feedback

\section{Introduction}

This paper presents the local tuning of a decentralised feedback control system, for vibration control of a plate, in terms of the mechanical power absorbed by the control units, and the electrical power supplied. Experimental results, which are validated by numerical simulations, demonstrate the local tuning of the velocity feedback units through power absorption maximisation for a two-channel control system. Moreover, the results obtained are contrasted with the minimisation of the global vibration level; and the electrical power, required by the two units, is investigated as a measure of the control effort of the control system.

\footnotetext{
${ }^{*}$ Corresponding author

Email address: s. camperi@soton. ac.uk (Stefano Camperi)
} 
In active structural control, the design of the controller is a crucial part, as it defines how the actuators are driven, according to the information from the sensors. This is often posed in terms of optimising certain control objectives with respect to specific constraints. In the case of vibration control of lightweight plates with low internal damping, the control objective is usually expressed as the minimisation of the global vibration level, and is quantified by its kinetic energy, for example. The constraint, on the other hand, is the minimisation of the implementation costs, as well as the computational requirements.

In fully centralised architectures, the information from the sensors converges to a single controller. The kinetic energy can be easily estimated, and very effective control laws can be implemented to target specific modes [1], or to design an internal model control [2]. However, this solution can be expensive because of cabling and implementation, since many sensors are required to be connected to the central controller. In addition, the complexity of the control law, as well as the data processing at each time sample, is computationally demanding. Finally, the control system is not robust to the failure of each sensor, and the mismatch between the controller model and the actual plant may lead to an increase in the level of vibration.

For the aforementioned reasons, several papers [3-5] have focused on moving towards decentralised architectures, in which part of the control action is carried out by sub-controllers, which operate locally. An example of this approach is the high-authority/low-authority control (HAC/LAC) system, in which the low-authority controller adds active damping through a simple local velocity feedback, and the high-authority controller accounts for the global behaviour of the structure [6].

In the extreme case, a fully decentralised control system can be thought, in which independent control units, consisting of a collocated sensor and actuator, act independently on the plate [5,7-10]. This is a rather appealing architecture, since it enables the design of a robust control system based upon self-contained modular units, where the complexity of the control design does not scale with the number of units implemented.

There are some concerns, though, on the performance of a fully decentralised architecture. In this regard, Baumann et al. [3] have shown that in some cases a fully decentralised velocity feedback control system can provide a global reduction of the kinetic energy comparable with the one obtained from a fully centralised control system. This result suggested that vibration reduction can be obtained by introducing active damping with local velocity feedback units, which are robust and can be implemented in a decentralised arrangement.

The challenge in this solution is the tuning of the control units, in order to determine the feedback gains. Although the minimisation of the kinetic energy guarantees a global reduction of vibration, a large number of sensors is required to obtain an accurate estimation of the kinetic energy. This poses a limit in terms of costs for the control system, as well as in terms of the design of a decentralised control system. On the other hand, the minimisation of the local velocity of the plate at the control position can converge to a pinning condition [9], in which the local velocity of the plate tends to zero, but the global vibration is not minimised.

In order to perform the tuning of the control unit locally, Zilletti et al. [9] considered the local mechanical power absorbed by the control unit from the structure with ideal force actuators, and showed that it almost corresponds to the minimisation of the kinetic energy $[9,11]$.

Previous studies $[9,11]$ have investigated the reduction of kinetic energy and the mechanical power absorbed with respect to the velocity feedback gains, when two control units with reactive actuators are implemented. Although this control system is unconditionally stable, the reactive actuators require an external support to react off, which may be impractical for real applications.

In this work, inertial actuators are considered, and their dynamic behaviour is taken into account to assess the conditional stability of the control system. This is due to the phase shift of the control force generated by an inertial actuator below its first natural frequency. In the literature, several methods have been suggested to improve the stability of a direct velocity feedback with inertial actuators. Examples of these studies include inerter elements [12] and compensator filters [13] to lower the natural frequency of the inertial actuator. Other studies focused on making 
the control system perfectly collocated by introducing a pair of poles and zeros in the open-loop transfer function by adding a feed-through term to the feedback loop [14] or an additional filter [15]. However, in this study it is shown that, if a suitable inertial actuator is used, with high internal damping and a low natural frequency, the global level of vibration of a plate can be minimised with a simple direct velocity feedback.

In this paper, the tuning of a decentralised velocity feedback control system with two control units is extended to the case in which inertial actuators are used. The dynamic behaviour of each inertial actuator is described in terms of their electrical and mechanical impedances, with respect to the base velocity at the control position on the plate, and to the control current. This enables us to estimate the mechanical and electrical power absorbed by each unit, directly from the velocity signal and a model of the inertial actuators only.

Moreover, the analysis of the kinetic energy with respect to the feedback gains is suitable for the tuning of the control units, but having some drawbacks. First, it does not contain information about the control effort, since it does not directly relate to the feedback gains. Second, this approach is not suitable for the implementation of additional control units, and it does not allow comparison between different types of actuators, or control positions.

The novelty of this work is to measure the reduction of kinetic energy of the plate with respect to the electrical power consumed by the control system, rather than to the feedback gains. The advantage of this approach is that different solutions can be easily compared (such as control systems with different number of units, actuators, or control positions) in a meaningful way.

The contribution of this paper is organised in two parts. First, for the tuning of the control units, the maximisation of the total mechanical power absorbed by the control system is shown to correspond to the minimisation of the kinetic energy, and the optimal gain for each unit is observed to be independent from the feedback gain implemented on the other unit. Second, for the efficiency of the control system, the two-channel arrangement is found to outperform the single-channel one, not only for vibration reduction, but also for the required electrical power, which is almost the same as the single unit. This implies that the two-channel control system corresponds to a more efficient distribution of the control effort among the units.

The paper is structured in 5 sections. In Section 2, a frequency-domain formulation of the the electrical and mechanical power absorbed by the inertial actuator from the plate is introduced as a function of the actuator impedance and plate velocity at the control position. In Section 3, the experimental setup is described, as well as the digital control system and the objectives. In Section 4, the stability of the feedback control system is discussed. In Section 5, the performance of the control system is investigated, when only one unit at the time is active. Finally, in Section 6 , the tuning and performance of a decentralised control system with two control units is discussed.

\section{Mathematical model}

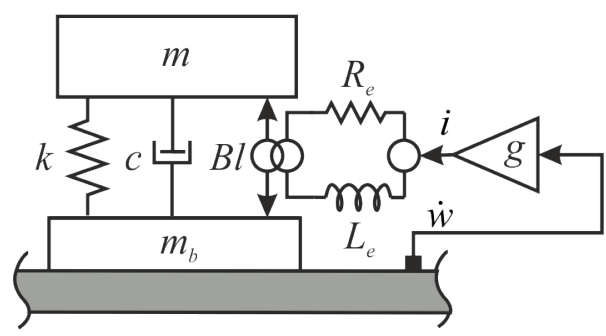

Figure 1: Schematic representation of a velocity feedback control unit with inertial actuator.

In this section, a decentralised velocity feedback control system with inertial actuators on a plate is considered. Each unit consists of a collocated velocity sensor and inertial actuator, which is driven with a current proportional to 
the local velocity of the plate, as shown in Fig. 1. The objective of this section is to derive a formulation for the power flow and kinetic energy of the plate, which does not require an identification of the plate dynamics. In order to do that, the dynamic behaviour of the inertial actuator is considered.

The power flow into the structure can be expressed in terms of the actuator dynamics, which can be conveniently described in the frequency domain as a two-port network [16], where the control force $f_{c}$ and the voltage $e$ are expressed with respect to the base velocity $\dot{w}$ and the current $i$ input, as,

$$
\left\{\begin{array}{c}
f_{c}=T_{i} i-Z_{m o} \dot{w} \\
e=Z_{e b} i+T_{i} \dot{w}
\end{array},\right.
$$

where $Z_{m o}$ is the open-loop mechanical impedance of the inertial actuator for a base excitation, $Z_{e b}$ is the blocked electrical impedance seen by the electrical system when a current excitation is present, and $T_{i}$ is the transduction function of the force transmitted to the ground generated by a current flowing, or, equivalently, an induced voltage generated by the base movement. At low frequencies, for a linear system, the inertial actuator can be described as a rigid suspended magnet of mass $m$. By writing the dynamic problem associated with the control system shown in Fig. 1, the complex frequency-dependent functions in Eq. (1) are equal to

$$
\begin{gathered}
Z_{m o}(\omega)=j m_{b} \omega+\frac{j m \omega(k+j c \omega)}{k+j c \omega-m \omega^{2}}, \\
T_{i}(\omega)=\frac{-m B l \omega^{2}}{k+j c \omega-m \omega^{2}}, \\
Z_{e b}(\omega)=R_{e}+j L_{e} \omega+\frac{j(B l)^{2} \omega}{k+j c \omega-m \omega^{2}},
\end{gathered}
$$

where $\omega$ is the frequency; $m_{b}, k, c$ are the base mass, and the stiffness and damping of the moving parts, respectively; $R_{e}$ and $L_{e}$ are the electrical resistance and inductance of the coil, respectively; $B$ is the flux density of the magnet and $l$ is the length of the coil.

When the control units are mounted on the plate, each actuator generates a control force on the plate, and thus it exchanges power. If the feedback gain is zero, the actuator absorbs power from the plate because of the passive coupling. However, as the feedback gain is increased, the electrical part of the inertial actuator contributes to the power flow between plate and actuator, through the force per unit current $T_{i}$ of Eq. (1). For a stationary and ergodic process, the mechanical power absorbed by each actuator from the plate can be written as the product between the control force $f_{c}$ and the plate velocity $\dot{w}$ as $[8,17,18]$

$$
\bar{P}_{m}=-E\left[\mathfrak{R}\left\{f_{c}^{H} \dot{w}\right\}\right],
$$

where $E$ is the expectation operator, $H$ indicates the complex conjugated, and $\mathfrak{R}$ the real part. The mechanical power flow between each control unit and the plate consists of a real and imaginary part. This is due to the phase lag between the control force and the plate velocity, which depends on the actuator dynamics. Reactive terms in the actuator generate a control force at the interface in quadrature with the velocity of the plate, and thus the power flow is imaginary. This means that the reactive power is continuously exchanged between the actuators and the plate, with no dissipation, and the net power flow is equal to zero [19]. Examples of these reactive terms are stiffness and mass elements on the mechanical side, and inductance terms on the electrical side. Conversely, dissipative elements generate a control force in opposite phase with the velocity of the plate, which generates a net power flow. Examples of dissipative terms are damping elements on the mechanical side, and resistance terms on the electrical side. Since this study is concerned with generating active damping, the analysis focuses on the mechanical power dissipated, and thus only the real part in Eq. (5) is considered. According to the sign convention adopted in Eq. (1) and in the scheme shown in Fig. 1, a minus sign is present in Eq. (5): positive values of mechanical power absorbed indicate a positive flow of power from the plate to the actuator. 
In the decentralised control system considered, the current in each actuator is proportional to the local velocity of the structure,

$$
i=-g \dot{w},
$$

where $g$ is the velocity feedback gain of the control unit considered.

From Eqs. (1) and (6), the mechanical power absorbed from Eq. (5) can be rewritten in terms of the local velocity of the structure and the actuator dynamics only, as [20]

$$
\bar{P}_{m}=E\left[\Re\left\{Z_{m o}+g T_{i}\right\}|\dot{w}|^{2}\right] .
$$

Furthermore, the electrical power supplied to each actuator can be measured, as the product of the current $i$ and the voltage $e$

$$
\bar{P}_{e l}=\left[\Re\left\{e^{H} i\right\}\right] .
$$

The number of the measured signals for each unit can be reduced through the estimation of the electrical power supplied as a function of the actuator dynamics and the velocity signal used for the feedback control, leading to:

$$
\bar{P}_{e l}=E\left[\Re\left\{Z_{e b} g^{2}-g T_{i}\right\}|\dot{w}|^{2}\right] .
$$

It is important to notice that, according to Eqs. (7) and (9), the power flow in the control system can be estimated locally, through the dynamic properties of the actuator, and the velocity of the plate, which is used for the feedback control. In case of a decentralised control system, in which several control units are mounted on the plate, the total mechanical and electrical powers can be measured as sums of the local powers of each unit.

The kinetic energy of the plate can be approximated as a summation of the squared values of the plate velocities at different locations on the plate [9]. The accuracy of the estimation depends on the number of points considered, and on the location. In the frequency domain, the kinetic energy $\bar{K}_{e}$ can be defined as integral of the Power Spectral Density (PSD) of the kinetic energy $S_{K}$ as [8]

$$
\bar{K}_{e}=\frac{1}{2 \pi} \int_{-\infty}^{\infty} S_{K} d \omega
$$

in which the PSD of the kinetic energy can be approximated as

$$
S_{K} \approx \frac{1}{2} m_{p} \sum_{i=1}^{M}\left|\dot{w}_{i}\right|^{2},
$$

where $M$ is the number of points where the plate velocities are measured, and $m_{p}$ is the mass of the plate. In this study, nine monitoring positions are considered, which provide a good estimate of the global level of vibration of the plate at low-frequency range, as confirmed by previous studies $[9,11,20]$.

\section{Experimental setup}

The objective of the experiment is to implement and analyse a decentralised velocity feedback control system on a plate with inertial actuators. The experimental setup for this study consists of a fully clamped, $2 \mathrm{~mm}$ thick, aluminium plate with two independent control units. The plate is mounted on a clamping frame specifically designed to avoid dynamic coupling with the structure [7], as shown in Fig. 2.

The decentralised feedback control system can be described as two independent control units, consisting of an accelerometer and an inertial actuator. The velocity feedback law from Eq. (6) can be rewritten for a two-channel decentralised feedback control system as

$$
\left\{\begin{array}{l}
i_{1} \\
i_{2}
\end{array}\right\}=\left[\begin{array}{cc}
g_{1} & 0 \\
0 & g_{2}
\end{array}\right]\left\{\begin{array}{l}
\dot{w}_{1} \\
\dot{w}_{2}
\end{array}\right\}
$$




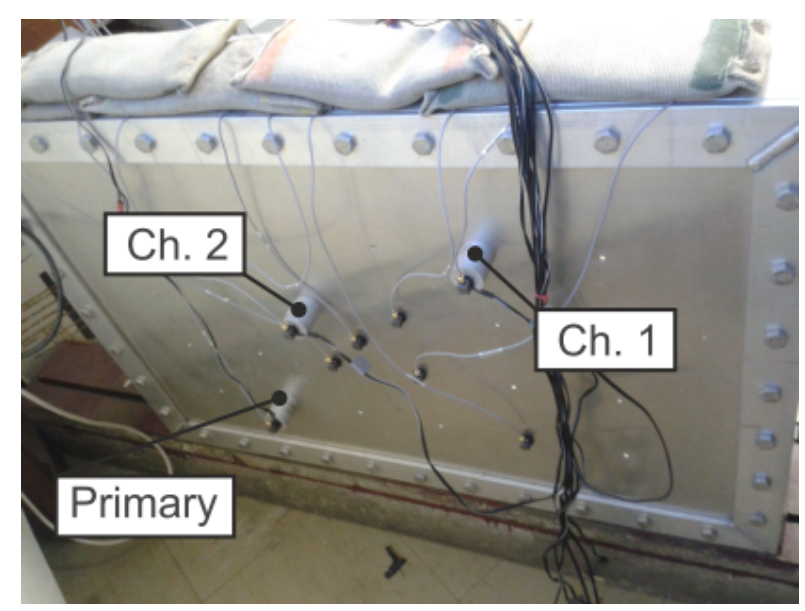

(a)

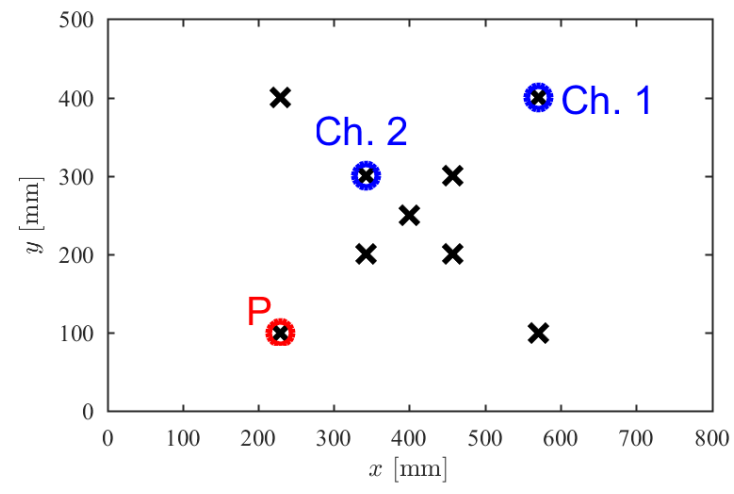

(b)

Figure 2: (a) Experimental setup and (b) schematic representation of the plate with a primary disturbance (P), two control units (Channel 1 and Channel 2), and nine accelerometers marked with (x).

in which the current $i_{n}$ in the $n$-th control unit is proportional to the local velocity of the plate $\dot{w}_{n}$.

In this experiment, the kinetic energy and the power flow are investigated with respect to 441 different pairs of feedback gains $\left(g_{1}, g_{2}\right)$, generated by 21 different values of $g_{1}$ and $g_{2}$.

The plate is excited by a point primary disturbance, and seven additional accelerometers are used to monitor the global vibration level. The physical and geometric properties of the experimental setup are summarised in Table 1 .

In order to replicate a more realistic case study, in which a number of different primary sources of disturbance act on a structure, the position of the primary excitation on the plate has been chosen in such a way to avoid nodal lines associated with the first few modes of the structure. In this way the low order modes are excited, and the action of the active control on these modes can be observed. For similar reasons, the two control units have been mounted to avoid nodal lines and symmetric positions. Furthermore, the distance between the primary disturbance and the control units has been taken into account. The objective of the experiment is to investigate the tuning of a control system subjected to unknown broadband disturbance. For this reason, a sensible choice is to avoid control positions too close to the primary excitation. In the extreme case, in which the control unit is collocated with the primary disturbance, the optimal control corresponds to pin the plate, so that no power is injected into the structure by the disturbance. However, as the control unit is moved away from the primary disturbance, the optimal control increasingly depends on the dynamic properties of the structure only, and the results are more significant for generic applications, in which a number of vibration sources, or a distributed disturbance, may be present. A more detailed discussion on the position of the control units is provided in Appendix Appendix A. Finally, the kinetic energy is estimated according to Eq. (10), through the velocity of the plate measured at nine accelerometers positions, i.e. the seven monitoring accelerometers and the two used for the feedback control by the control units. The number and position of the nine monitoring accelerometers B\&K4507-B-004 have been chosen in such a way to guarantee an accurate estimation of the kinetic energy in the frequency range of interest, up to $1 \mathrm{kHz}$.

The digital control system, and the measure of the kinetic energy and of the power flow, is performed with a $\mathrm{dSPACE}^{\circledR}$ DS1 103 PPC Control Board, running with a sampling frequency $f_{s}$ of $16 \mathrm{kHz}$, which is configured through the dSPACE ControlDesk ${ }^{\circledR}$ and Matlab Simulink ${ }^{\circledR}$ software, and summarised in Fig. 3.

In each control unit, the measured local acceleration of the structure is read by the dSPACE ${ }^{\circledR}$ Control Board, and digitally integrated to obtain the local velocity. Two independent current signals are then generated, proportional to the respective velocities by means of two feedback gains. The current signals are then filtered with a low-pass filter $\mathrm{KEMO} \mathrm{VBF} / 8$ to avoid aliasing and reduce spillover at high frequencies, and sent to a custom designed 4-channel 


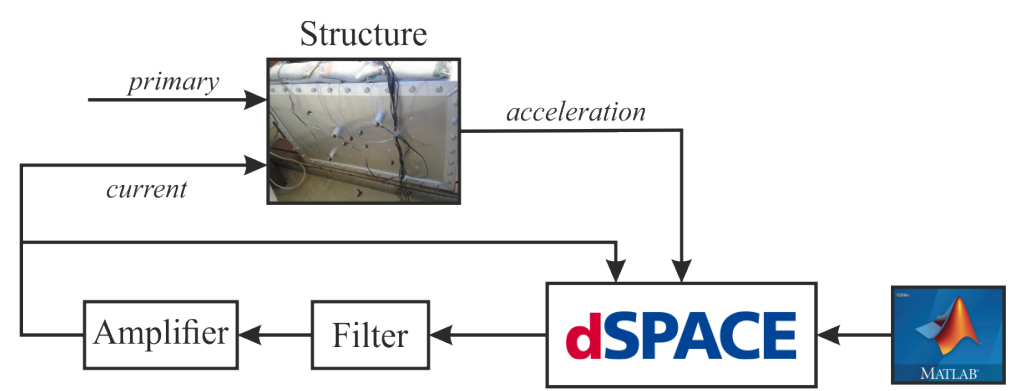

Figure 3: Schematic representation of the digital control scheme.

Micromega current amplifier, which drives the Micromega IA01 inertial actuator.

In parallel to the two independent control channels, the velocity of the plate is acquired from the nine accelerometers to estimate the kinetic energy. Moreover, a digital twin of the inertial actuator is implemented, through three digital filters, to measure the power flow according to Eqs. (7) and (9). Each filter replicates one of the three impedances of the inertial actuator from Eq. (1), so that the control force and the voltage of each actuator can be estimated directly from the local velocity of the structure, used for the feedback control.

Finally, the same dSPACE ${ }^{\circledR}$ Control Board is used to generate the primary disturbance and assess the stability of each unit. The primary disturbance is generated driving the primary actuator with a white noise, which is filtered with a low-pass elliptic filter KEMO VBF/8, with cut-off frequency of $1 \mathrm{kHz}$. The primary disturbance signal generated by the Control Board is filtered with a low-pass elliptic filter KEMO VBF/8, with cut-off frequency of $1 \mathrm{kHz}$, to concentrate the energy in the frequency range below $1 \mathrm{kHz}$, and to excite the plate properly, without driving the primary actuator at high power levels. This, however, does not affect the spillover at high frequencies, which depends on the delay introduced to each control channel by the digital Control Board and the low-pass filter.

The experimental results are validated through an equivalent analytical mobility/impedance model [17], in which the plate is assumed to be fully clamped and the inertial actuator behaviour is described by the two-port network system presented in Eq. (1). 


\begin{tabular}{lll}
\hline & Parameter & Value \\
\hline Aluminium plate & Dimensions $\left(\mathrm{mm}^{2}\right)$ & $800 \mathrm{x} 500$ \\
& Thickness $(\mathrm{mm})$ & 2 \\
& Density $\left(\mathrm{kg} / \mathrm{m}^{3}\right)$ & 2720 \\
& Young's modulus $\left(\mathrm{N} / \mathrm{m}^{2}\right)$ & $7 \times 10^{10}$ \\
& Poisson ratio & 0.33 \\
& Internal damping ratio & $1 \%$ \\
& & \\
Sensors positions & Pos. \#1 $(x, y)(\mathrm{mm})$ - primary & $(229,100)$ \\
& Pos. \#2 $(x, y)(\mathrm{mm})$ & $(571,100)$ \\
& Pos. \#3 $(x, y)(\mathrm{mm})$ & $(343,200)$ \\
& Pos. \#4 $(x, y)(\mathrm{mm})$ & $(457,200)$ \\
& Pos. \#5 $(x, y)(\mathrm{mm})$ & $(400,250)$ \\
& Pos. \#6 $(x, y)(\mathrm{mm})$ & $(457,300)$ \\
& Pos. \#7 $(x, y)(\mathrm{mm})-$ channel 2 & $(343,300)$ \\
& Pos. \#8 $(x, y)(\mathrm{mm})$ & $(229,400)$ \\
& Pos. \#9 $(x, y)(\mathrm{mm})-$ channel 1 & $(571,400)$ \\
& & \\
Micromega IA01 & Moving mass $(\mathrm{g})$ & 36 \\
& Base mass $(\mathrm{g})$ & 53 \\
& Natural frequency $(\mathrm{Hz})$ & 8.7 \\
& Damping ratio & 0.3 \\
& Voice coil coefficient $(\mathrm{N} / \mathrm{A})$ & 1.6 \\
& Electrical resistance $(\Omega)$ & 3 \\
& Electrical inductance $(\mu \mathrm{H})$ & 100 \\
\hline
\end{tabular}

Table 1: Geometry and physical properties of the experimental setup shown in Fig. 2.

\section{Stability analysis}

The stability margin of each unit can be assessed, according to the Nyquist Criterion, measuring the open-loop transfer function between an independent input signal to the actuator and the collocated measured velocity. However, the gain margin on each unit depends on the feedback gain implemented on the other unit. In particular, previous studies $[3,10]$ have shown that the stability margin of each unit, in a decentralised velocity feedback control system, decreases as the feedback gain in the other units increases.

In this study, the stability analysis of the two-channel control system is performed in two steps. First the stability margin of each unit is determined for the single-channel configurations, in which only one control unit is operating, while the other one is switched off. Second, for the two-channel control system, a grid of feedback gain pairs is defined, and the stability of each configuration is investigated.

For the two-channel feedback control system, the velocity of the plate $\dot{\mathbf{w}}_{\mathbf{c}}$ at the control positions, according to

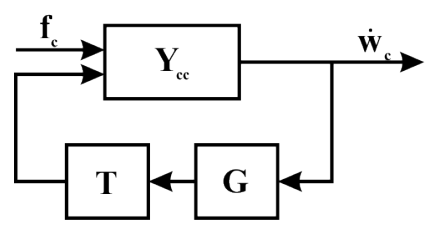

Figure 4: Block diagram of the decentralised velocity feedback control system. 
Fig. 4, is equal to

$$
\dot{\mathbf{w}}_{\mathbf{c}}=\left[\mathbf{I}+\mathbf{Y}_{\mathrm{cc}} \mathbf{T G}\right]^{-1} \mathbf{Y}_{\mathrm{cc}} \mathbf{f}_{\mathbf{c}},
$$

where $\mathbf{f}_{\mathbf{c}}$ is the primary disturbance; $\mathbf{I}$ is the identity matrix; $\mathbf{Y}_{\mathbf{c c}}$ the plate point-mobility matrix of the plate between the sensors and actuators positions; and $\mathbf{T}$ and $\mathbf{G}$ are diagonal matrices consisting of, respectively, the force per unit current and the feedback gains of each channel.

According to the Generalised Nyquist Criterion [21], the stability of the two-channel feedback control system in Eq. (13) is guaranteed if the locus of the function

$$
\operatorname{det}\left[\mathbf{I}+\mathbf{Y}_{\mathbf{c c}} \mathbf{T G}\right]
$$

does not encircle the origin [21], where det is the determinant of a matrix.

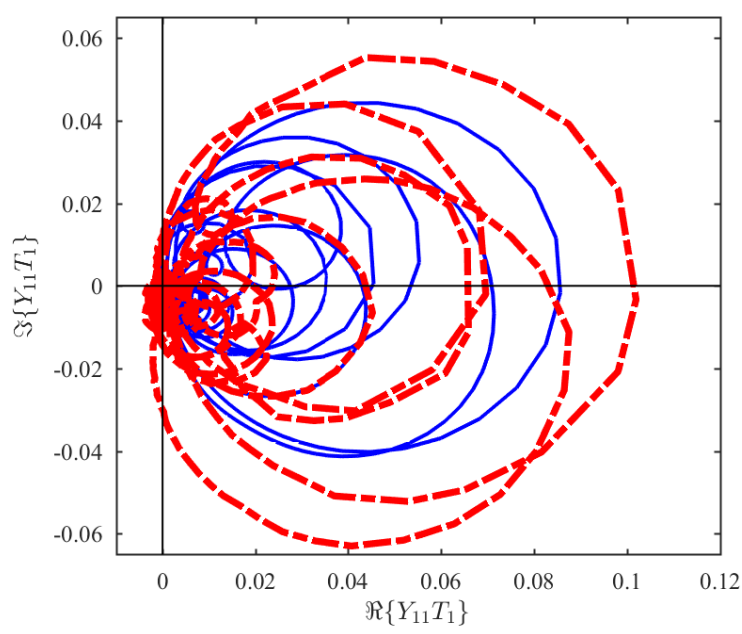

(a)
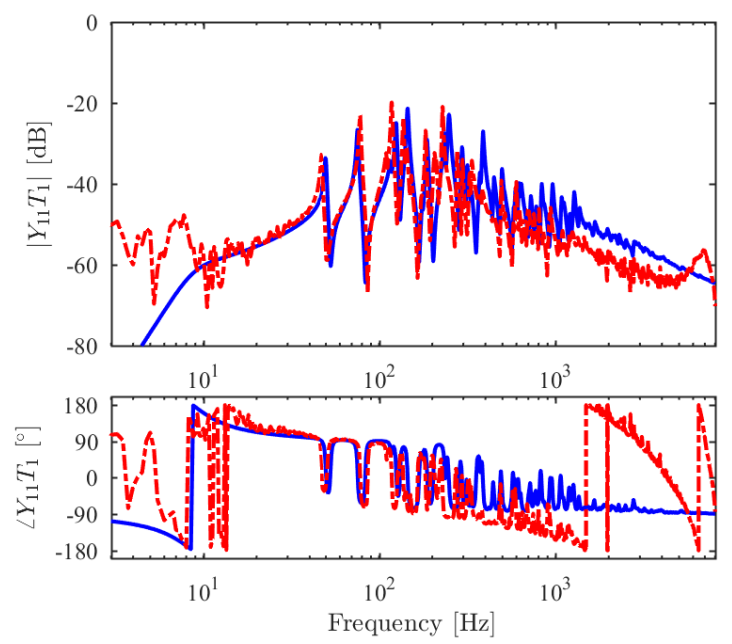

(c)

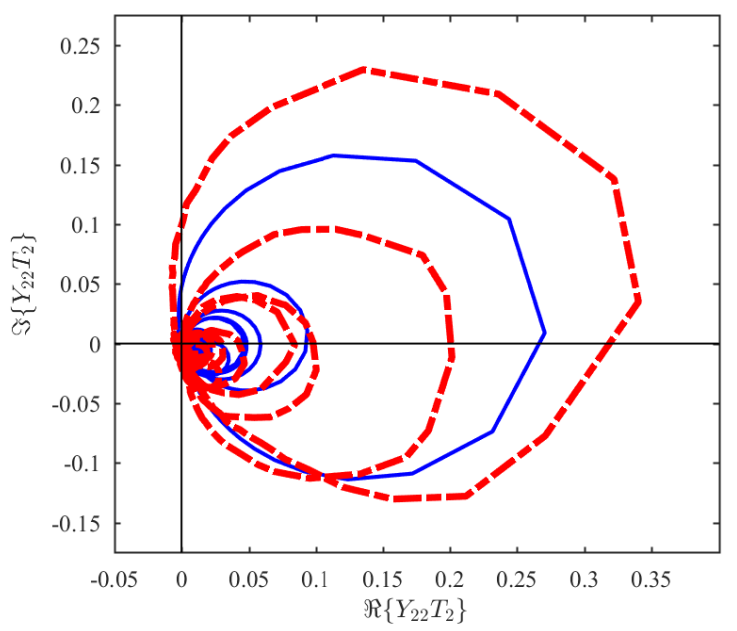

(b)
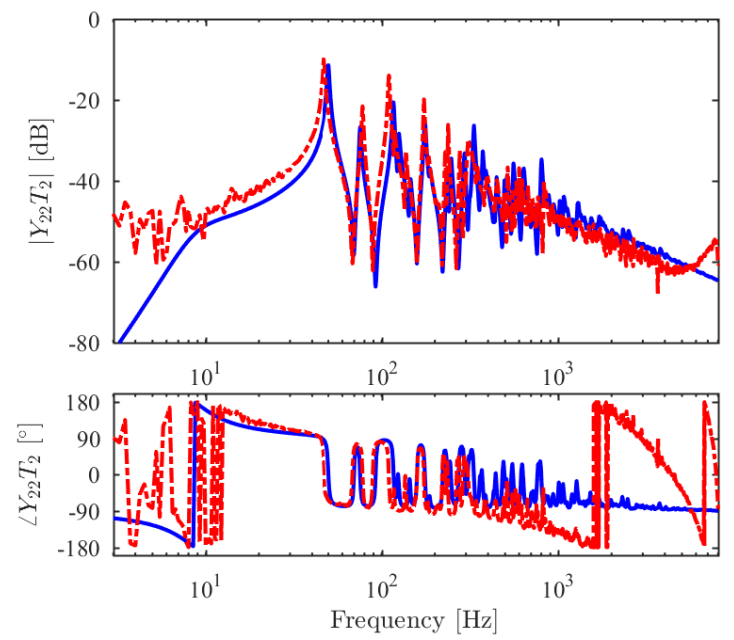

(d)

Figure 5: Nyquist plots of the (a) single channel 1 and (b) single channel 2, for a unitary value of feedback gain when the other channel is switched off, and respective Bode plots in (c) and (d). Numerical results in solid blue, measured results in dash-dotted red. (For interpretation to the references to colour in this figure, the reader is referred to the web version of this article.) 
When the single-channel case is considered, the locus in Eq. (14) reduces to the transfer function between the input current in the actuator and the output velocity of the plate at the actuator position. In this case, according to the Nyquist Criterion, the maximum stable gain of the single-channel control system can be measured, as the limit value for which the open-loop transfer function encircles the point $(-1,0)$ in the Nyquist plot. The Nyquist and Bode plots of the single-channel control systems are shown in Fig. 5. Since, on each channel, actuator and sensor are collocated, the real part of the open-loop transfer function is positive at almost all frequencies, providing good stability properties. However, because of the dynamic coupling of the inertial actuator with the plate, a shift in phase of $180^{\circ}$ occurs below the natural frequency of the inertial actuator, around $10 \mathrm{~Hz}$, which makes the control system only conditionally stable, as shown in Fig. 5c and Fig. 5d.

Moreover, the amplitude of the circles on the positive part of the real axis is proportional to the amount of damping which can be introduced in each mode. For example, the second channel, in Fig. 5b, is located in a more central position on the plate with respect to the first channel, and thus it is coupled more effectively with the first structural mode of the plate. As a consequence, a larger amount of damping can be introduced in the first mode; but, on the other hand, the gain margin is smaller. The maximum stable gain, on each channel, is found to be equal to, respectively, $650 \mathrm{As} / \mathrm{m}$ and $210 \mathrm{As} / \mathrm{m}$.

In the mathematical model, a number of assumptions have been made. First the amplifier has been assumed to be ideal and equal to a fixed gain. Second the anti-aliasing filter and the digital processing have not been considered, and the plate has been assumed to be homogeneous and fully clamped. As a result, the mathematical model overestimates the stability of the control system, especially because of the delay at high frequencies, and the noise in the measurements at low frequencies. The numerical maximum stable gains are equal to $1280 \mathrm{As} / \mathrm{m}$ and $450 \mathrm{As} / \mathrm{m}$, respectively. However, the model provides results in reasonable agreement with the experiments and correctly describes the relevant dynamic properties of the control system, and thus it can be used to give an insight into the problem studied.

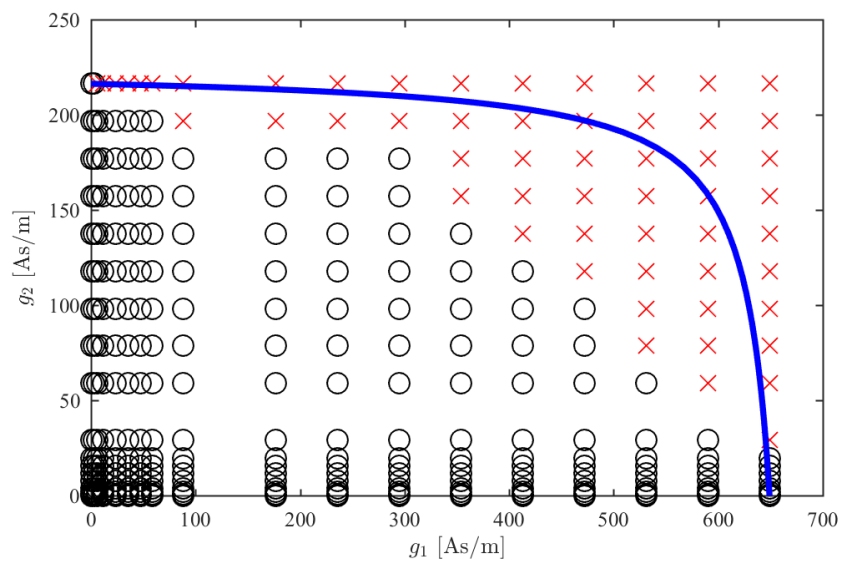

Figure 6: Stability region of the two-channel control system, and grid of the 441 measured couples of feedback gains. Black circle marks indicate experimental stable feedback gain pairs, red cross marks indicate experimental unstable pairs. The solid blue line shows the numerical stability limit, scaled with respect to the maximum stable gains found experimentally for the single-channel cases. (For interpretation to the references to colour in this figure, the reader is referred to the web version of this article.)

Once the maximum stable gains are obtained, a grid of 441 feedback gain pairs can be defined, as a combination of 21 different feedback gains on each channel, ranging from zero up to the respective maximum stable gain. The grid of feedback gains is shown in Fig. 6.

In order to investigate the stability of the two-channel control system, a sequential loop closing technique has been adopted, in which the feedback gain on the first channel is kept constant, and the feedback gain on the second channel is increased up to an unstable configuration. The result is a stability region, shown in Fig. 6, in which the twochannel control system is stable. Because of the difference between the maximum stable gains obtained from the measurements and the numerical analysis, the blue line in Fig. 6 has been scaled to match the experimental maximum 


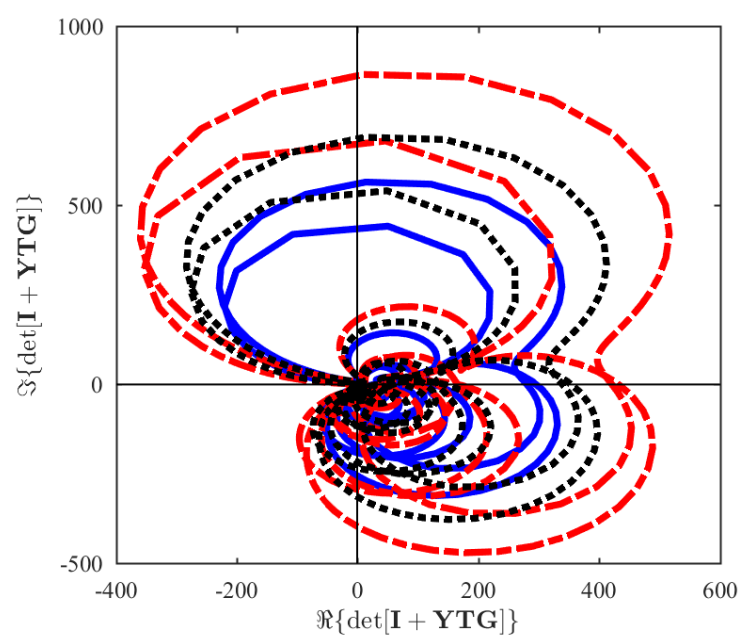

(a)

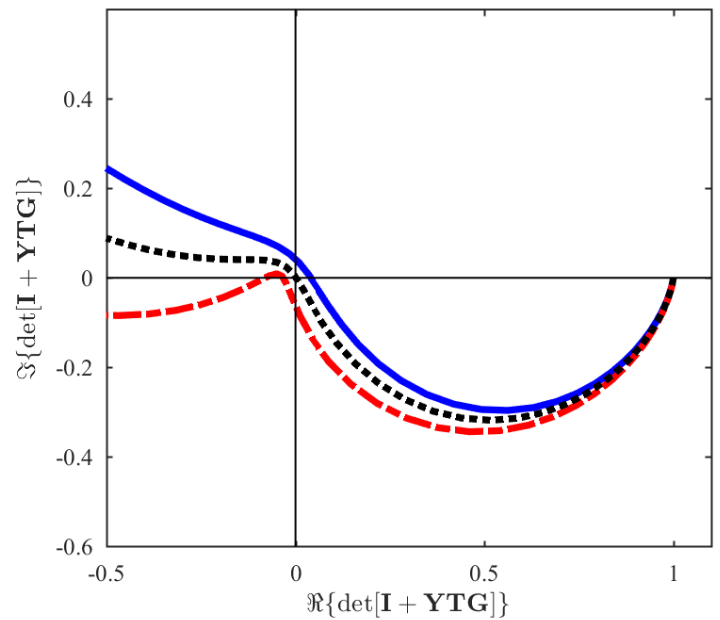

(b)

Figure 7: Locus of the function det $\left[\mathbf{I}+\mathbf{Y}_{\mathbf{c c}} \mathbf{T G}\right]$ for different feedback gain pairs $\left(g_{1}, g_{2}\right)$. Stable configuration in solid blue for the normalised gain pair equal to $(854,168)$, unstable configuration in dash-dotted red for the normalised gain pair equal to $(1024,216)$, limit stable configuration in dotted black for the normalised gain pair equal to $(939,188)$. (a) Global view, (b) Zoomed view. (For interpretation to the references to colour in this figure, the reader is referred to the web version of this article.)

feedback gains found in the two single-channel configurations.

The distance of each point in Fig. 6 from the blue line provides a measure of the stability margin of that specific configuration. In particular, for a given value of feedback gain in one of the two control units, the stability margin of the global control system reduces if the feedback gain in the other channel is increased. This means that any feedback gain pair, in a decentralised velocity control system, corresponds to a gain margin smaller than the one obtained with only one of the two active channels. This proves the robustness of the control system subject to failures of a control unit since, in case one of the two control units fails, the gain margin of the control system increases.

The stability region obtained with the sequential loop closing in Fig. 6 can be further validated by applying the Generalised Nyquist Criterion from Eq. (14) to the two-channel control system, as shown in Fig. 7. For stable feedback gain pairs the function does not encircle the origin, and the system is stable. However, for larger feedback gain pairs the function encircles the origin and the system is unstable.

\section{Single-channel control system}

In this section, the changes of kinetic energy and power flow into the system, when a single control unit is tuned, are investigated.

In order to evaluate the effect of each unit, only one channel at a time is kept active while the other one acts passively on the structure, i.e. without any feedback gain implemented. This means that, from Eq. (12), $g_{2}$ is kept equal to zero, while the performance of channel 1 is assessed, and vice-versa.

The main focus of this section is to determine the performance of the single control unit as a measure of comparison for the two-channel control system as discussed in Section 6.

First an analysis in the frequency domain is carried out to assess the effectiveness of the active damping, and to determine the frequency region of interest [20]. Finally, the performance of the control system is investigated with respect to the feedback gain and the electrical power. 


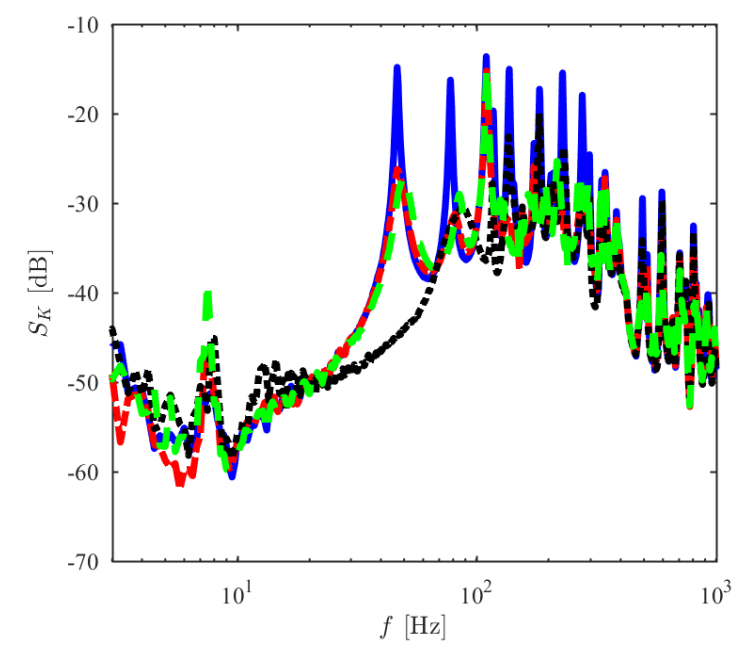

(a)

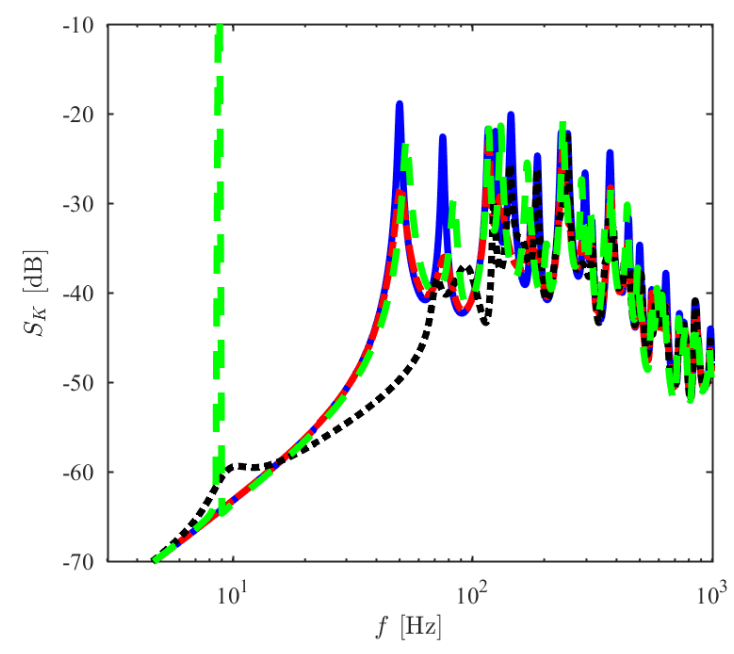

(b)

Figure 8: PSD of the kinetic energy of the plate, estimated with 9 accelerometers, (a) measured and (b) simulated for: the uncontrolled configuration (solid blue); only channel 1 active and optimally tuned (dash-dotted red); only channel 2 active and optimally tuned (dotted black); and only channel 1 active and close to instability (dashed green). (For interpretation to the references to colour in this figure, the reader is referred to the web version of this article.)

\subsection{Spectral analysis}

The local velocity feedback control introduces active damping into the plate. The amount of damping introduced in each mode depends on the value of the feedback gain, as well as the position of the control unit. The global effect of the active damping action can be assessed from the total kinetic energy, which can be obtained as the integral of the PSD of the kinetic energy in the frequency domain. However, in order to have a more clear estimation of the damping effect, it is important to determine the frequency region in which a significant reduction of vibration can be achieved [17].

The reduction of kinetic energy in the frequency domain, with respect to different values of feedback gain, is shown in Fig. 8 and the simulated and experimental results are compared. The uncontrolled plate response (solid blue line) is mostly determined by its resonant terms. Since the position of the primary excitation has been chosen in such a way to avoid nodal lines, all the first structural modes are excited. The red dashed line shows the reduction of kinetic energy when the first channel is optimally tuned, and the second channel is switched off. It can be noticed that active damping is effectively introduced in all the first six structural modes, except for the third mode. This is due to the fact that the control position is close to a nodal line of the third mode. Conversely, the black dotted line shows the effect of the second channel, when the first one is not active. In this case, because the control position is close to the centre of the plate, a larger reduction can be achieved in the first structural mode, up to $40 \mathrm{~dB}$. However, this channel is less effective for higher modes, such as the fifth and the sixth modes, since their nodal lines are close to the centre of the plate. Finally the green dashed line shows the PSD of the kinetic energy of the plate when the feedback gain of the first channel approaches the maximum stable gain. In this case, the kinetic energy is not minimised any more, because a large control force is generated by the control unit, which almost pins the plate at the control position. This is equivalent to changing the boundary conditions of the plate, and it is confirmed by a new set of structural resonances appearing at higher frequencies. This can be observed for example, in the measurements in Fig. 8a for the second structural mode at $85 \mathrm{~Hz}$. At the same time, because of the phase shift in the control force below the actuator resonant frequency, a new peak rises at the actuator resonance around $9 \mathrm{~Hz}$, and eventually approaches infinite amplitude of vibration for the unstable configuration. However, in the experiments, this is limited by the stroke saturation of the inertial actuator.

The experimental and simulated results differ in a number of aspects. First, below the first structural resonance, 
noise is present in the measurements. Secondly, a shift in resonance is present, due to the non-ideal boundary conditions of the experimental setup, which is assumed to be fully clamped in the simulations. Finally, sensors and actuators are not perfectly collocated in the experimental setup, and this affects the performance of the control system, especially at high frequencies above $500 \mathrm{~Hz}$. However, although these differences exist, the model can still correctly describe the experimental results, and it can be used for comparison purposes.

According to the effect of the active damping of the two channels in the frequency domain, the estimation of the total kinetic energy, and the power flow, is limited to the frequency range $10-200 \mathrm{~Hz}$, since active damping is particularly effective within this range. The frequency region below $10 \mathrm{~Hz}$ has not been included because of the noise at low frequencies in the experiments. However, during the analysis of the results, different frequency ranges of integration have also been tried, such as 0-200 Hz. However, no significant variations in the results were observed because the changes in the noise at low frequencies for different values of feedback gain were found to be small, with respect to the changes around the structural resonances.

\subsection{Kinetic energy and Power flow}

The performance of the two control units with respect to the feedback gains is analysed, in terms of the kinetic energy and the power flow, which are obtained as the integral of the PSDs in the frequency domain, up to $200 \mathrm{~Hz}$.

The kinetic energy gives an estimation of the global reduction of vibration in the plate. Conversely, the power flow is investigated for a number of reasons: to validate the measure of the mechanical power absorbed from the plate as a local way of tuning the feedback gain; and to measure the electrical power required by the control unit, which represents the control effort.

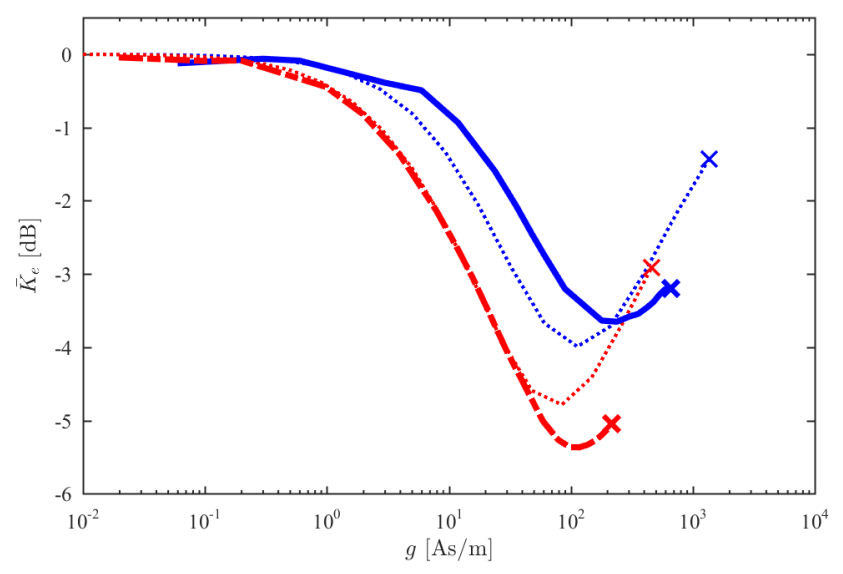

Figure 9: Time-averaged kinetic energy of the plate as integral of the PSD of the kinetic energy in the frequency range 10-200 Hz with respect to the feedback gain, for a single channel velocity feedback control. Channel 1 in solid blue, channel 2 in dash-dotted red. Measured results in thick lines, simulated in thin dotted lines. Cross marks indicates maximum stable gains. (For interpretation to the references to colour in this figure, the reader is referred to the web version of this article.)

The reduction of kinetic energy obtained by using either one of the two channels is shown in Fig. 9, with respect to the relative feedback gain. The measured and simulated results with respect to the feedback gains are in good agreement. This suggests that, although the plate dynamics has not been correctly identified (as discussed in Fig. 8), the mathematical model is accurate enough to describe the relevant properties of the control system. If the channel one is considered (solid blue line), as the feedback gain $g_{1}$ increases, the kinetic energy reduces down to $-4 \mathrm{~dB}$. For further increase in the feedback gain, the level of vibration rises again, because a pinning condition is approached $[17,18,22]$. However, the pinning condition is not achieved, because of the conditional stability of the feedback control system. This is due to the control force generated by the inertial actuator, which has a shift of $180^{\circ}$ in phase below its first resonance, which is around $9 \mathrm{~Hz}$ and makes the real part of the plant response to be negative [23]. The second channel (dashed red line) shows similar performance. However, because the second channel is closer to the 
centre of the plate, more damping can be introduced in the first structural mode, resulting in a global reduction of kinetic energy equal to $5 \mathrm{~dB}$. Though, the greater coupling of the second control unit with the first structural mode, makes the second channel less stable, having a lower gain margin. Finally, the experimental setup is less stable than what expected from the mathematical model. This is due to the noise at low frequency, where the instability occurs, and to the spillover effect at high frequencies due to the delay introduced into the control loop by the digital control system, which have not been included in the mathematical model.

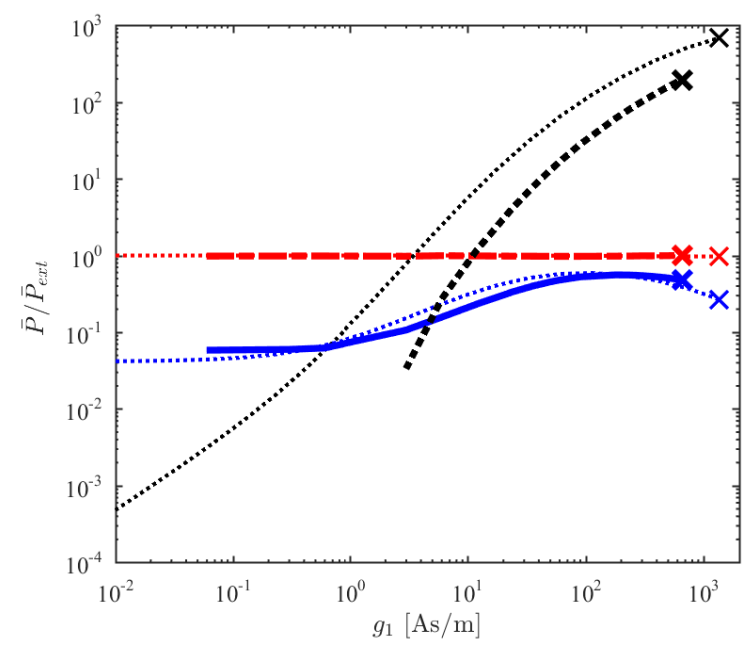

(a)

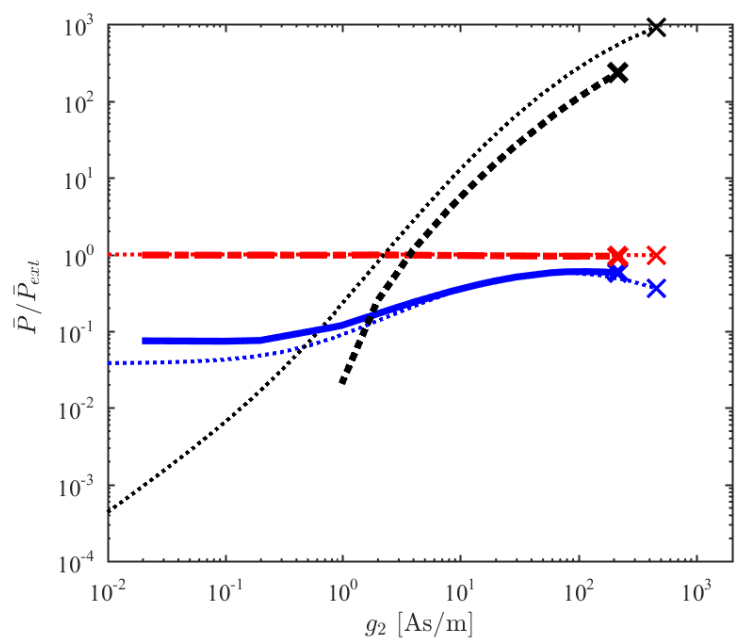

(b)

Figure 10: Power flow with respect to the individual feedback gain for (a) channel 1, and (b) channel 2 in the frequency range 10-200 Hz. Mechanical power absorbed by the control unit from the structure in solid blue, external power from the primary disturbance in dash-dotted red, and electrical power supplied by the control unit in dotted black. Measured results in thick lines, simulated in thin dotted lines. Power normalised with respect to the maximum external power. Cross marks indicates maximum stable gains. (For interpretation to the references to colour in this figure, the reader is referred to the web version of this article.)

The power flow in the control system with respect to the feedback gain is shown, respectively, in Fig. 10a for the first channel, and in Fig. 10b for the second channel. The results are normalised according to the maximum external power (dashed red line), injected into the plate by the primary disturbance. The mechanical power absorbed by the inertial actuator from the structure (solid blue line) shows a trend similar to the kinetic energy. For low values of feedback gain, close to zero, the mechanical power absorbed corresponds to the passive coupling of the actuator with the plate, and it depends on the mechanical damping of the actuator and its position on the plate relative to the primary excitation. As the feedback gain increases, the mechanical power absorbed approaches a maximum, which corresponds to the minimum in the kinetic energy. However, for further increase in the feedback gain, the mechanical power absorbed reduces because of the pinning condition, in which the local velocity of the plate tends to zero. Therefore, the mechanical power absorbed can be used as a local index to tune the velocity feedback control unit $[17,22]$. The advantage of this solution is that a self-contained control unit can be designed, in which an array of sensors is no longer required for the tuning.

Moreover, from the power balance applied to the plate, if power losses through the boundary conditions are neglected, the difference between the external and the mechanical power absorbed by one channel is equal to the power internally dissipated by the plate and the power passively absorbed by the other channel. Therefore, when the optimal gain is implemented, the power internally dissipated by the plate is minimised, i.e. the transfer of power from the disturbance to the control unit is maximised. This represents an extension of the analytical study carried out in Ref. [24] for a single-degree-of-freedom structure to the case of a continuous structure, such as the plate considered.

Finally, the electrical power required by the control unit to generate the control force (dotted black line) grows constantly with the feedback gain. This trend is mostly determined by the electrical resistance of the actuator, and represents a direct measure of the control effort required to implement the control system. 


\section{Two-channel control system}

In this section, the effect of the two channels acting simultaneously on a decentralised arrangement is investigated. In such a control system, the two inertial actuators are driven with control currents proportional to the respective local velocities, by means of two independent feedback gains $g_{1}$ and $g_{2}$.

Therefore the analysis carried out on a single channel at the time in the previous section needs to be extended to the multidimensional case, in which the kinetic energy and the power flow are function of all the feedback gains. This corresponds to a huge increase in the complexity of the analysis, and therefore an alternative method to reduce this complexity is presented in this section considering two channels. The final objective is to contrast the benefit of two control units with the total electrical power.

The analysis is divided into three parts:

- Global performance. The reduction of kinetic energy and the total mechanical power absorbed by the two control units from the plate.

- Local performance. The local mechanical power absorbed by each control unit from the plate and the electrical power.

- Control effort. The reduction of kinetic energy with respect to the total electrical power.

The objective of the first part is to validate the maximisation of the mechanical power absorbed for the tuning of the control units, as an alternative solution to the kinetic energy minimisation for the two-channel control system. In the second part, the local power flow on each unit is investigated to assess the cross-interaction between different control units. Finally, in the third part, the performance of the two-channel decentralised control system is contrasted with the single-channel case.

The results are obtained implementing 441 different pairs of feedback gains $\left(g_{1}, g_{2}\right)$, in which 21 different values of $g_{1}$ and $g_{2}$ are considered ranging from 0 to $220 \mathrm{As} / \mathrm{m}$.

\subsection{Global performance}

The reduction of kinetic energy and the total mechanical power absorbed by the two control units from the plate with respect to two feedback gains, $g_{1}$ and $g_{2}$, are shown in Fig. 11. In particular, on the left-hand side, Fig. 11a and Fig. 11c, the experimental results are shown, while on the right-hand side the relative simulation results are depicted. The kinetic energy has a global minimum, where a reduction of vibration of more than $8 \mathrm{~dB}$ is achieved. This means that the individual performance of the control units almost sums up, providing a reduction of kinetic energy almost equivalent to the sum of the single reductions obtained by each channel in Section 5. Moreover, the optimal gain pair $\left(g_{1}, g_{2}\right)$, minimising the kinetic energy, is equivalent to the optimal gains, $g_{1}$ and $g_{2}$, found for the single-channel cases in the previous section. This means that the optimal gain for one channel does not depend on the gain implemented in the other channel. This is a rather important result, which is in agreement with previous studies on the tuning of decentralised velocity feedback control systems [8,11,25]. Gardonio et al. [8] obtained similar results when studying a decentralised velocity feedback control system with ideal force actuators. In their study, they analysed the distance between different control units and found differences in the optimal gains only when the distance was smaller than the smaller wavelength considered in the numerical model.

The total mechanical power absorbed by the two control units from the plate has a global maximum, which corresponds to the minimum in the kinetic energy. This is a rather important result, as it shows that a sequential loop closing can be implemented, in which the global synchronisation between different units suggested by Zilletti et al. $[9,11]$ is greatly simplified. In a sequential loop closing arrangement, the tuning of each channel can be performed in series since the tuning of one channel does not detune other channels, and the convergence of the algorithm to the global minimum is guaranteed.

Furthermore, these results are particularly relevant for the robustness of the control system, even in the case of failure of a control unit, since the other one is still optimally tuned and stable. 


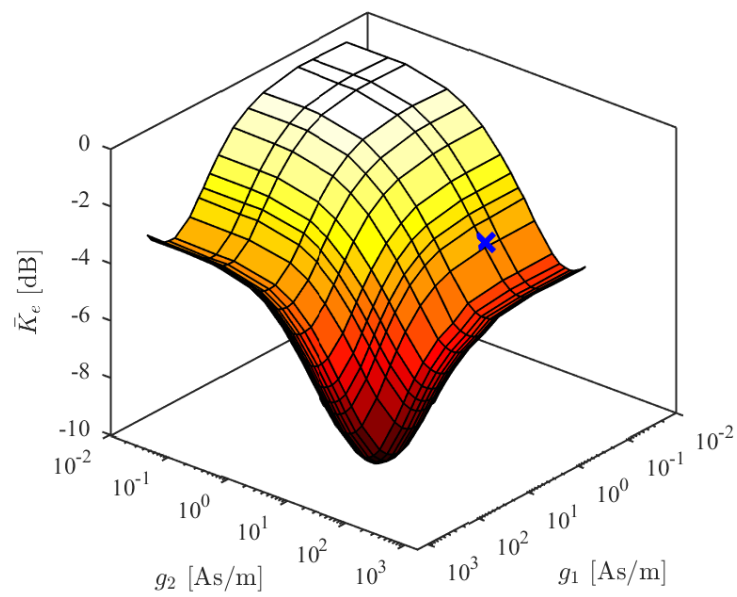

(a)

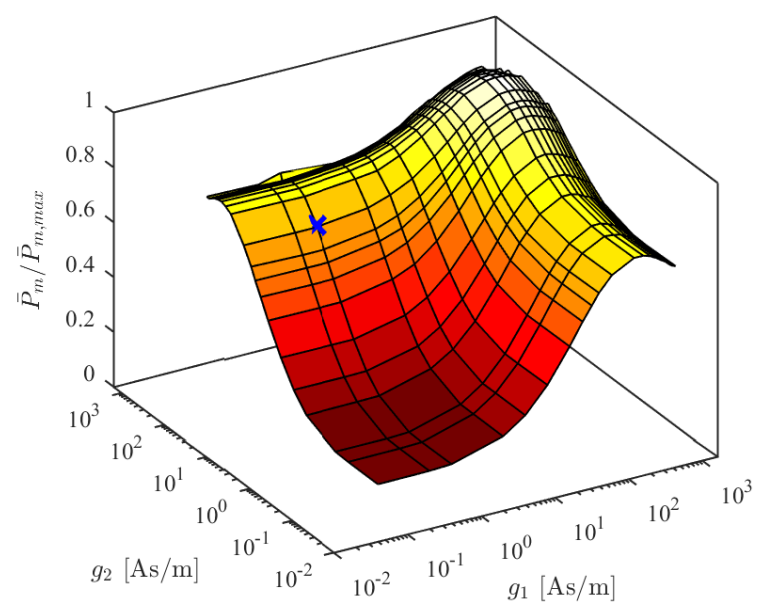

(c)

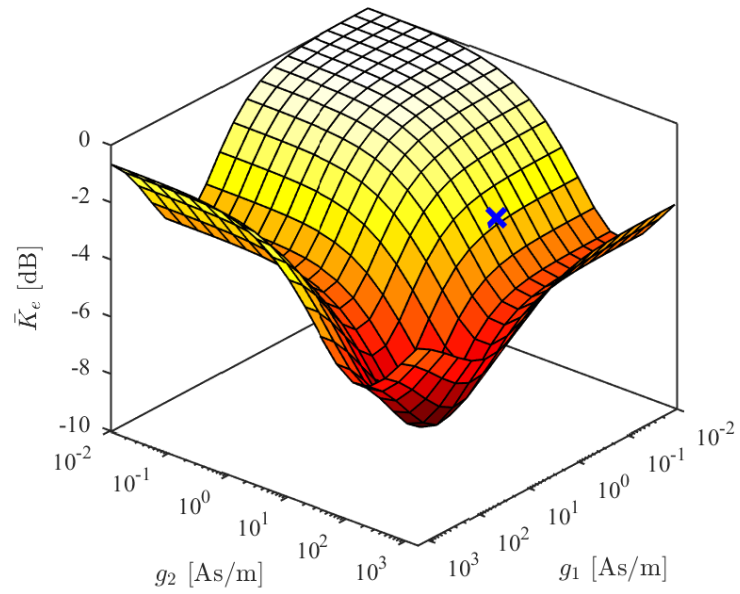

(b)

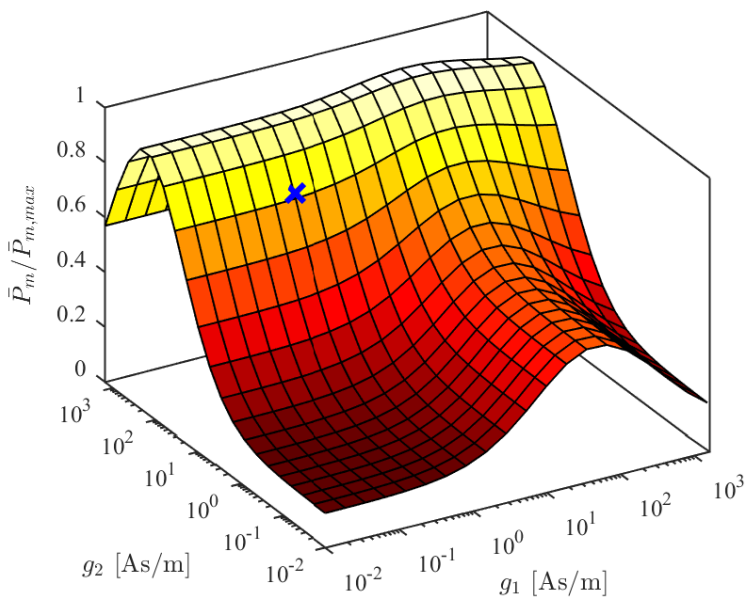

(d)

Figure 11: $(a, b)$ Kinetic energy of the plate, and (c, d) total mechanical power absorbed by the control units from the structure, with respect to the feedback gains $g_{1}$ and $g_{2}$ in the frequency range 10-200 Hz. Measured results in (a, c), and simulated results in (b, d). For better readability, different views are adopted for the kinetic energy and the total mechanical power absorbed. In order to make the comparison easier, a blue cross reference point is introduced.

\subsection{Local performance}

The sequential loop closing discussed in the previous subsection requires the measure of the total mechanical power absorbed by both control units, which means that communication between the single units has to occur. In this subsection, the local power of each control unit is considered independently.

The local powers absorbed by the first and second unit, with respect to the feedback gains $g_{1}$ and $g_{2}$, are shown in Fig. 12. The powers are normalised with respect to the maximum total mechanical power absorbed. The experimental results on the right-hand side, in Fig. 12a for the first channel and in Fig. 12c for the second channel, are in good agreement with the relative numerical results, respectively in Fig. 12b and Fig. 12d.

In order to analyse the effect of the two feedback gains, the mechanical power absorbed by the first unit is first investigated, from Fig. 12a and Fig. 12b. For each value of $g_{2}$, the mechanical power absorbed by the first channel with respect to $g_{1}$ shows the same trend observed for the single-channel case, with a maximum at the optimal values 
of $g_{1}$. Similarly, the mechanical power absorbed by the second unit shows a dual trend with respect to $g_{2}$. This shows that the tuning of each control unit can be performed locally in a sequential loop closing arrangement.

The electrical power required by the two control unit is shown in Fig. 13, with a comparison between experimental results on the left-hand side (Fig. 13a and Fig. 13c) and numerical results on the right hand side (Fig. 13b and Fig. 13d). The electrical power required by each unit largely depends on the respective feedback gain, and it is quite insensitive to the changes in the other feedback gain. However, it can be noticed that, the electrical power required by one unit slightly decreases as the feedback gain of the other unit increases. This depends on the cross-interaction between the two units, in which the control effort of a single unit decreases as the one on the other unit increases.

\subsection{Control effort}

The two-channel control system outperforms the single-channel one in terms of reduction of vibration. However, in an active control system, the control effort required to perform the control needs to be taken into account.

The solution proposed here, is to re-map the reduction of kinetic energy shown in Fig. 11 to the total electrical power required by the two control units, which corresponds to the sum of the single electrical powers shown in Fig. 13. This can be considered as an attempt to analyse the performance of the decentralised feedback control system in terms of reduction of vibration against control effort, which is typical of the optimal control theory [4].

The result is shown in Fig. 14, where each point has a size and colour proportional to the percentage of electrical power required by the first channel: this enables to analyse the distribution of the electrical power between the two different units.

It is significant to compare the results with the performance of the two single-channel arrangements from the first channel (solid blue) and the second channel (dashed blue). Although the two-channel arrangement outperforms the single-channel ones in terms of reduction of the kinetic energy, the control effort does not increases. This means that the two-channel control system reduces the vibration on the plate more efficiently, requiring the same electrical power to operate, but simply redistributed on two different units. 


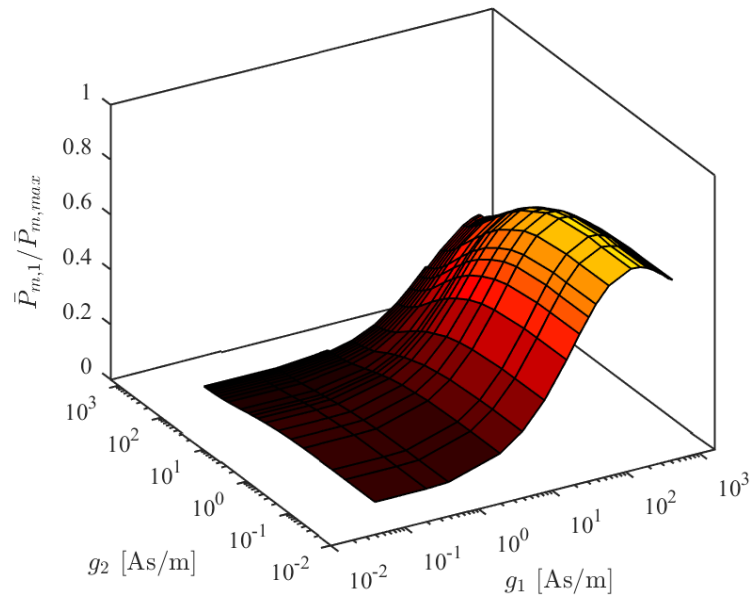

(a)

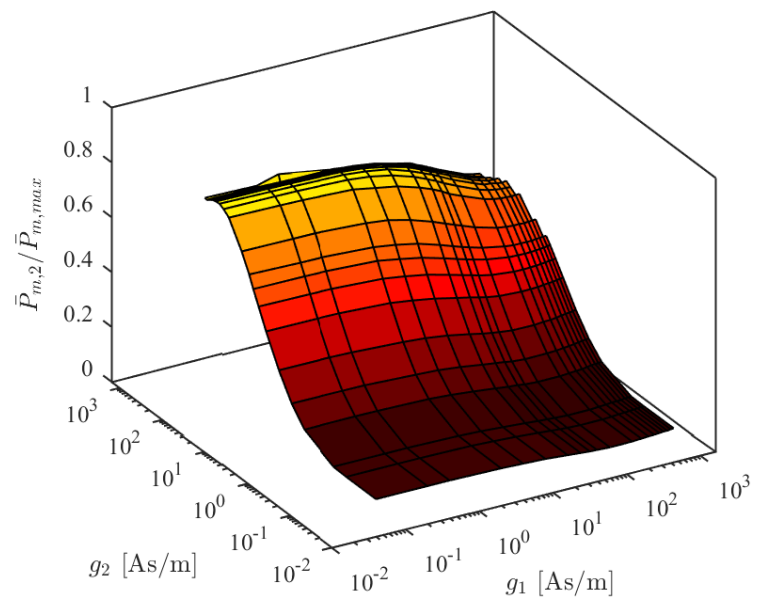

(c)

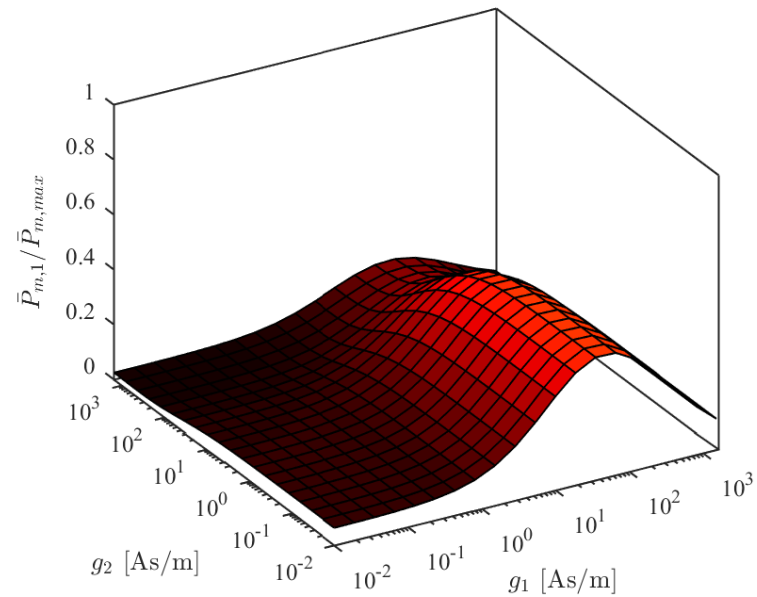

(b)

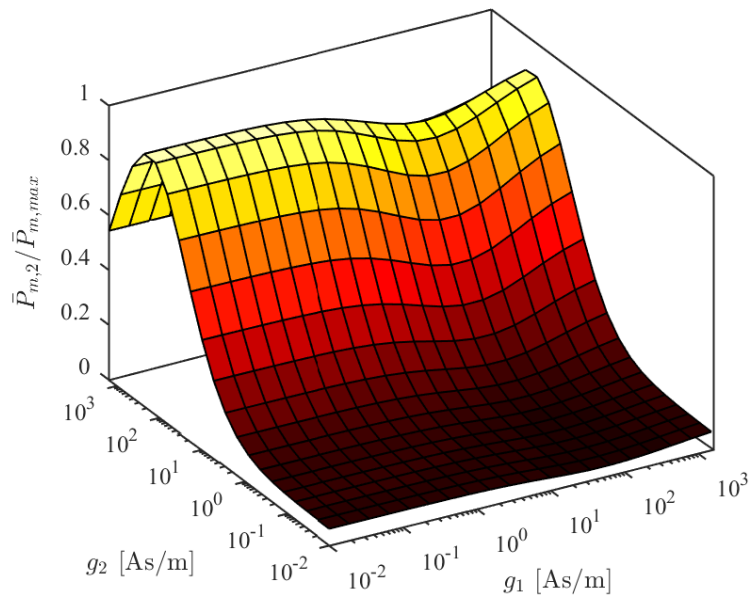

(d)

Figure 12: Mechanical power absorbed by (a, b) channel 1 and (c, d) channel 2 normalised with respect to the maximum total mechanical power absorbed, as function of the feedback gains $g_{1}$ and $g_{2}$ in the frequency range 10-200 Hz. Measured results in (a, c), and simulated results in (b, d). 


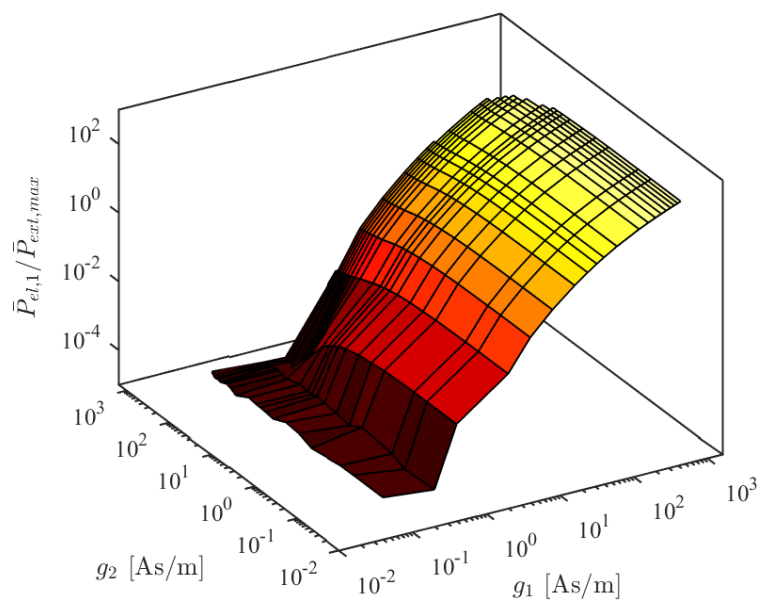

(a)

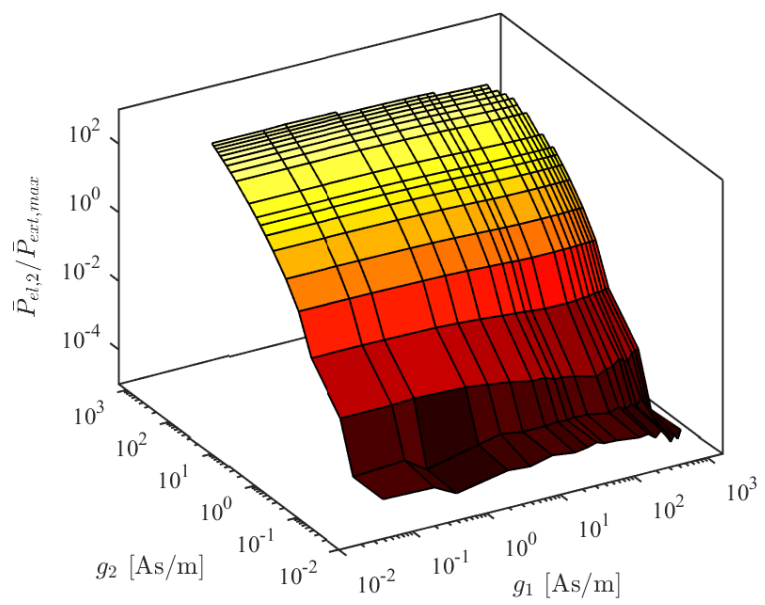

(c)

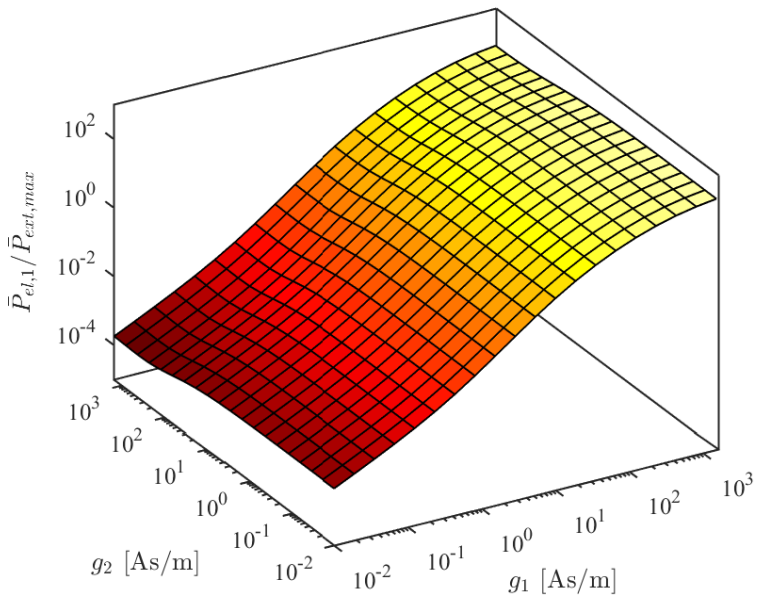

(b)

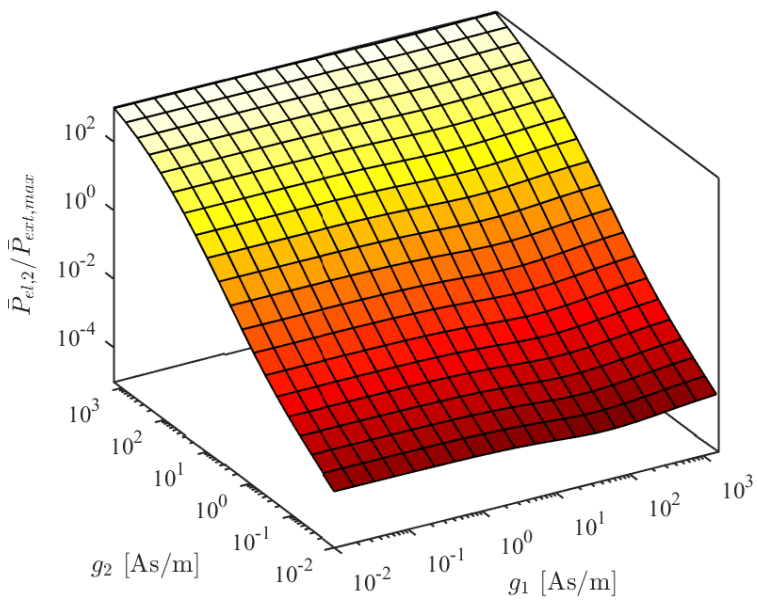

(d)

Figure 13: Electrical power by $(a, b)$ channel 1 and $(c, d)$ channel 2 normalised with respect to the external power from the primary, as function of the feedback gains $g_{1}$ and $g_{2}$ in the frequency range 10-200 Hz. Measured results in $(\mathrm{a}, \mathrm{c})$, and simulated results in $(\mathrm{b}, \mathrm{d})$. 


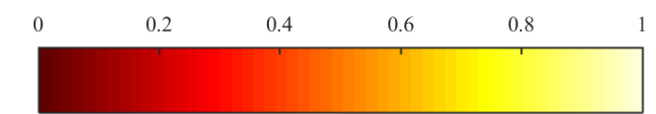

$\bar{P}_{e l, 1} / \bar{P}_{e l}$

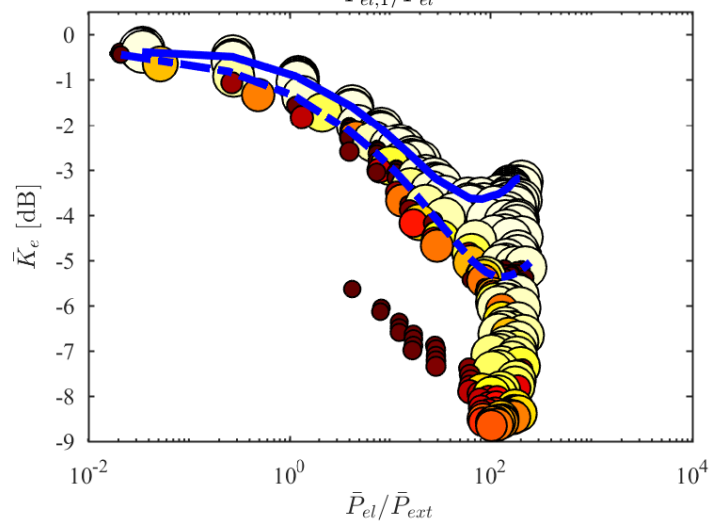

(a)

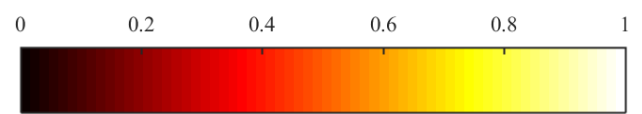

$\bar{P}_{e l, 1} / \bar{P}_{e l}$

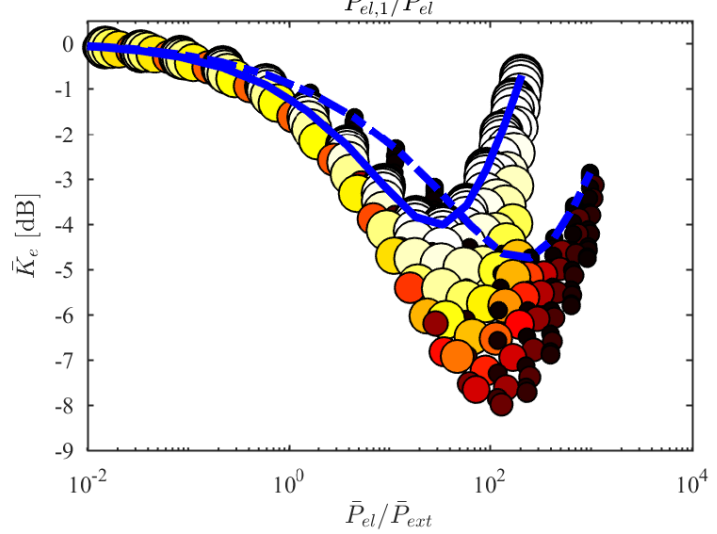

(b)

Figure 14: Kinetic energy of the plate (a) measured and (b) simulated with respect to the total electrical power, normalised with respect to the external power in the frequency range 10-200 Hz. The size and colour of each point is proportional to the percentage of electrical power provided to the channel 1. For comparison, the performance of the single channels are represented in solid blue (channel 1) and dash-dotted blue (channel 2). 


\section{Conclusions}

This paper presents the experimental and numerical tuning of a decentralised velocity feedback control system with inertial actuators for plate vibration. The study is enriched with an analysis on the power requirements of the active control, with respect to the reduction of vibration.

A straightforward formulation of the mechanical power absorbed from the structure and of the electrical power is provided, which depends exclusively on the dynamic properties of the inertial actuator and the local velocity of the structure, which is also used for the feedback control. This enables the design of a smart and self-contained control unit, which can be tuned without identifying the structure to be controlled.

The tuning of each control unit can be performed locally with respect to the mechanical power absorbed by the single unit, for one channel at the time, in a sequential loop closing fashion. The maximisation of the single powers absorbed by each unit converges to the global maximum of the total mechanical power absorbed by the control system from the structure, which corresponds to the minimum in the kinetic energy of the plate. This results is rather important, because it shows that the tuning of each unit is independent, and changes in the feedback gain in one channel does not detune the other control unit.

This is an extension of the work presented in Ref. [9], in which inertial actuators are used to perform the control, and their dynamic behaviour is included in the tuning.

In addition, the electrical power required by the inertial actuator to perform the control is introduced as a measure of the control effort. This enables to overcome some limitations of the analysis of the feedback control system just in terms of feedback gains. First, the electrical power provides a meaningful tool for a feasibility analysis of an active control system, as it enables to estimate the service costs. Second, it provides a valuable measure of comparison between different solutions in terms of number of control units used, type of actuators, and control locations. For example, the second control unit is the closest to the centre of the plate and therefore it can provide more reduction of vibration, mainly because it can easily control the first structural mode. However, it requires a larger amount of electrical power supplied, when compared to the first control unit. This suggests that the proposed control system does not require any particular study on the optimal position of the control unit, but only some intuitive considerations, such as to avoid the nodal lines of the first modes and control positions too close to fixed boundaries of the plate.

With reference to the study presented in this paper, the two-channel control system is proved not only to outperform the single-channel case providing more than $8 \mathrm{~dB}$ of global reduction of vibration, but is also competitive in terms of electrical power required. It is shown, indeed, that the optimal tuning corresponds to a configuration in which the amount of electrical power is comparable with the one of a single-channel case, but having a better distribution among the control units.

\section{Acknowledgements}

The authors gratefully acknowledge the European Commission for its support of the Marie Skłodowska-Curie program through the ITN ANTARES Project (GA 606817).

\section{Appendix A. Control unit position}

In this section the effect of the position of a single control unit on the plate is investigated, with respect to the plate dynamics and the disturbance source position. Intuitively, if a single point disturbance is present, great reductions of vibration can be achieved, if the primary force is completely cancelled out with a control force acting at the same position. However, if a more realistic case is considered, a number of different vibration sources is present on the plate, and a more general analysis is required.

This study is organised in two parts. First, a rain-on-the-roof excitation is considered, as worst case example, in which a continuous and uncorrelated distribution of random forces acts on the plate. Second, at the other extreme, a single point-force acting on the plate is analysed in order to highlight analogies and differences with the previous case, and to draw conclusions for the design of the experimental setup. In each case, the maximum reduction of the kinetic energy provided by the control unit, as well as the relative feedback gain, is analysed with respect to the control position $(x, y)$ on the plate. A similar study is carried out when the control unit is tuned to maximise the mechanical power 
absorption from the plate. The results are summarised in Figs. A.15 and A.16, where the kinetic energy is normalised with respect to the configuration with the feedback gain equal to zero; the absorbed power is normalised with respect to the maximum power absorbed; and the feedback gain is normalised with respect to the maximum stable gain.

In a rain-on-the-roof excitation, each structural mode is equally excited, and the energy is uniformly distributed throughout the plate. Thus the performance of the velocity feedback control unit depends uniquely on the coupling of the unit with the structural modes. As shown in Fig. A.15a, a larger broadband reduction of kinetic energy can be achieved when the nodal lines are avoided. It is important to notice that the configuration minimising the kinetic energy is always stable, with an optimal gain well below the maximum stable gain, as depicted in Fig. A.15b. However, as the control position approaches the centre of the plate, the optimal gain increases towards a less stable configuration. This is because the control unit dynamics increasingly couples with the first mode of the plate, reducing the maximum stability limit of the feedback control [23]. Nevertheless, for central positions, the broadband reduction of kinetic energy increasingly depends on the first structural mode only, since many of the higher modes have a node in the centre of the plate. This causes the optimal feedback gain to move towards higher gains, which correspond to a higher active damping of the first structural mode.

The maximisation of the mechanical power absorbed by the control unit gives similar results. As shown in Fig. A.15c, the positions more efficient in terms of power absorption correspond to those of Fig. A.15a, in which a large reduction of kinetic energy is achieved. Moreover, with respect to the tuning of the feedback gain, the maximisation of the mechanical power absorbed converges to values similar to those found in Fig. A.15b. Some differences can be noticed for central positions, in which the optimal gain from the power maximisation is lower than the one found from the kinetic energy. This is because, for central positions, the velocity of the plate increases in magnitude, and thus more power is passively absorbed by the control unit.

To summarise, if a structure is subjected to a rain-on-the-roof excitation, the performance of a local velocity feedback is maximised when the actuator couples with all the structural modes of interest. Furthermore, the tuning of the feedback gain, at any position, can be approximated with the maximisation of the mechanical power absorbed.

If a single point excitation is considered, the performance of the control system depends on the distance between the control unit and the primary excitation, as shown in Fig. A.16. As the control position approaches the disturbance, the control force cancels out the primary source more efficiently, and large reductions of vibration can be obtained up to $15 \mathrm{~dB}$, as shown in Fig. A.16a. The reduction of vibration, at this position, is only limited by the conditional stability of the control system, as confirmed in Fig. A.16b, in which the optimal gain converges to the maximum stable gain. This means that, in this configuration, the tuning of the control unit minimising the kinetic energy would result in an unstable control system.

However, as the distance from the primary disturbance increases, the reduction of kinetic energy increasingly depends on the dynamic properties of the plate, and similar conditions to those observed for the rain-on-the-roof excitation, in Fig. A.15a and Fig. A.15b, are observed.

Conversely, the maximisation of the power absorption provides a stable configuration at any control position, as shown in Fig. A.15c and Fig. A.15d. For large distances of the control unit from the primary excitation, the tuning of the control unit according to the mechanical power absorbed provides results similar to the kinetic energy minimisation, and to the rain-on-the-roof excitation. Moreover, as the control unit approaches the primary disturbance, the tuning of the mechanical power absorbed converges to low values of feedback gain, similar to those found for the rain-on-theroof excitation in Fig. A.15d. This is because large values of feedback gain would result in pinning the plate at the excitation position, reducing the mechanical power absorbed to zero. 

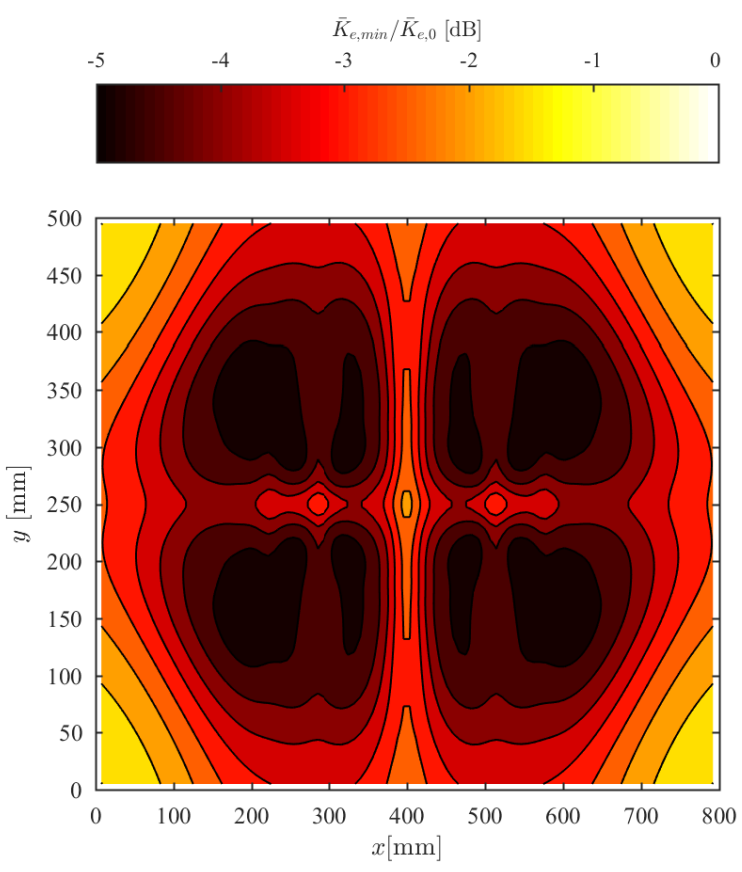

(a)
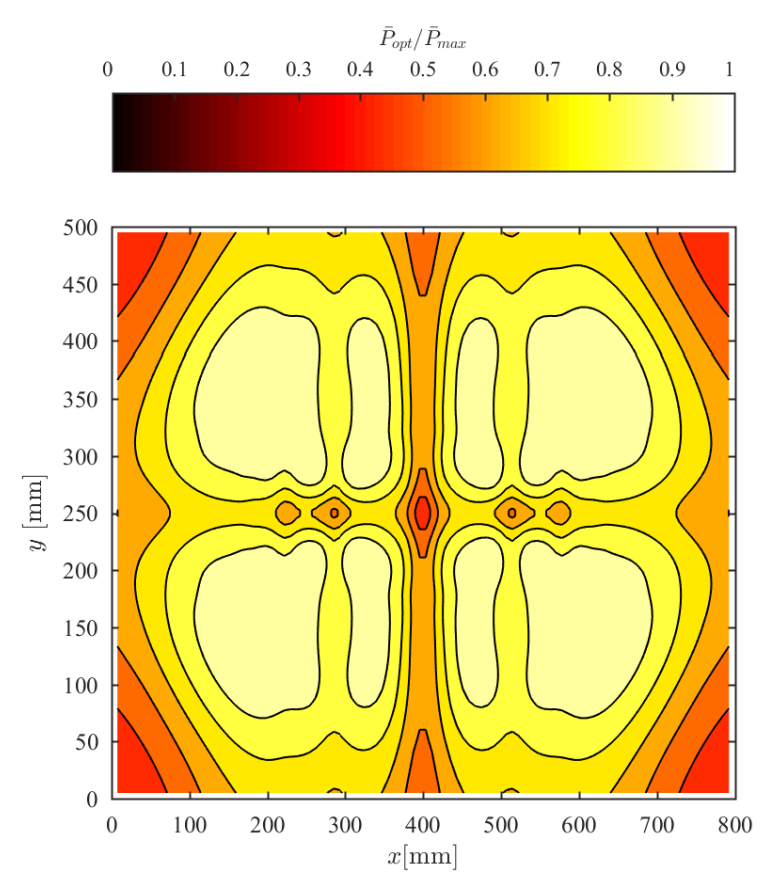

(c)
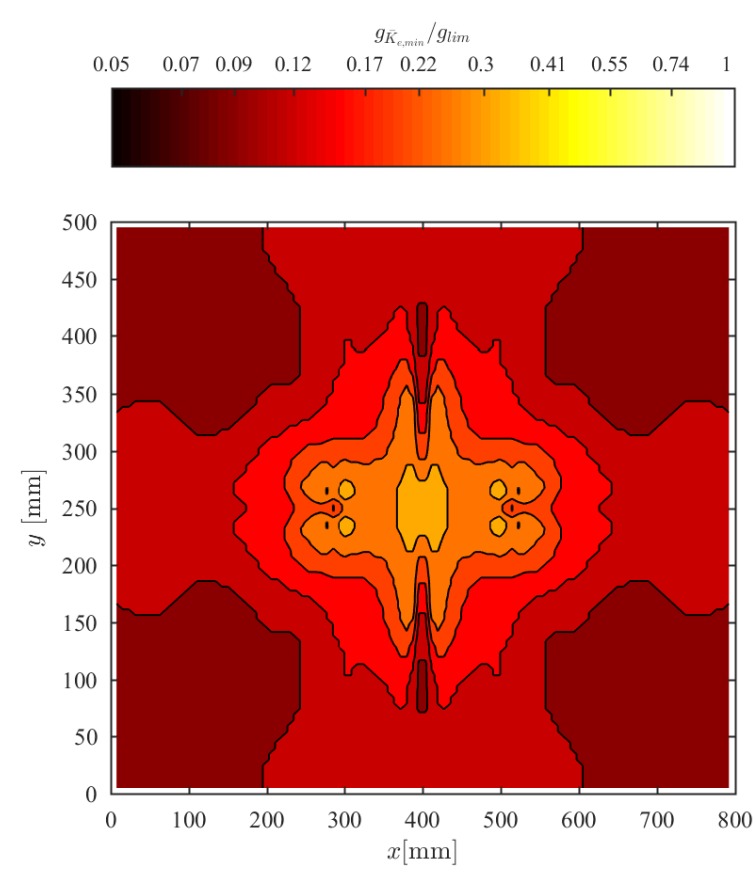

(b)

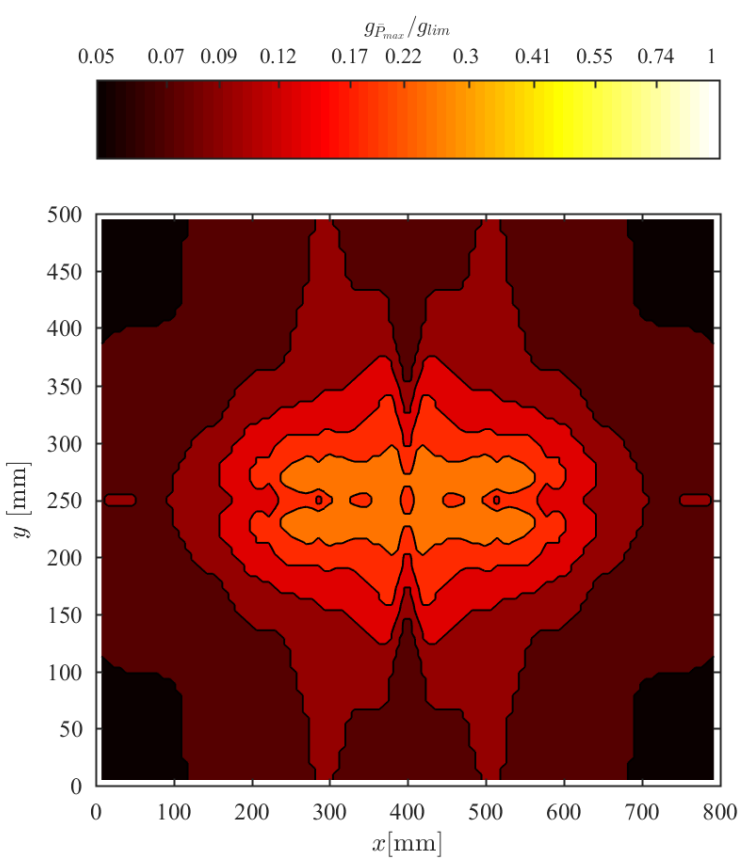

(d)

Figure A.15: Performance of a control unit at different position on a plate subjected to rain-on-the-roof excitation. (a) Minimum kinetic energy; (b) optimal gain minimising the kinetic energy; (c) maximum mechanical power absorbed by the control unit from the structure; (d) optimal gain maximising the mechanical power absorbed. Frequency range $0-200 \mathrm{~Hz}$. 

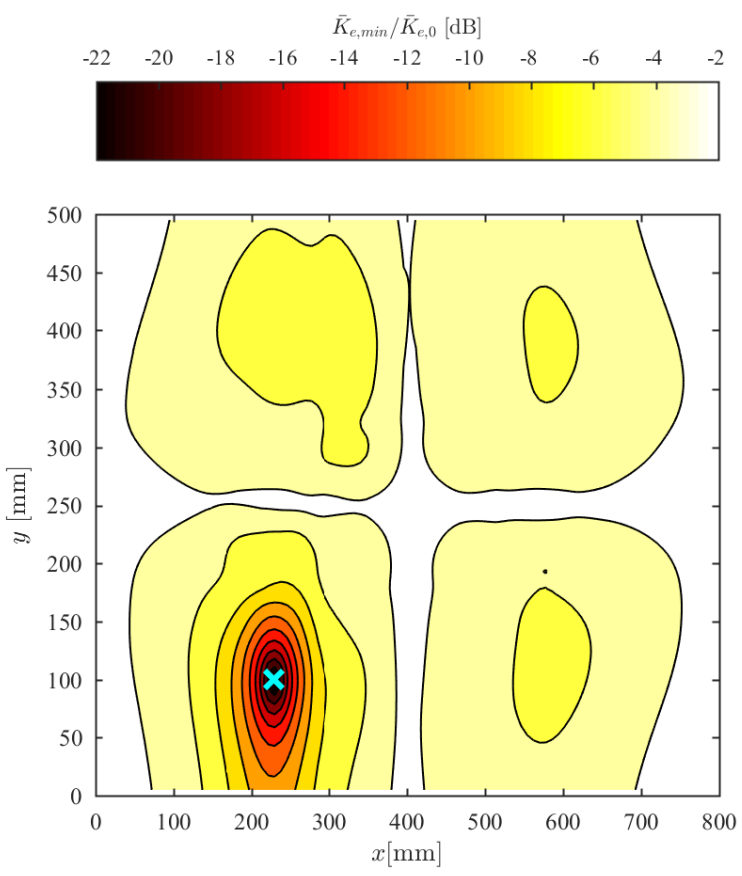

(a)

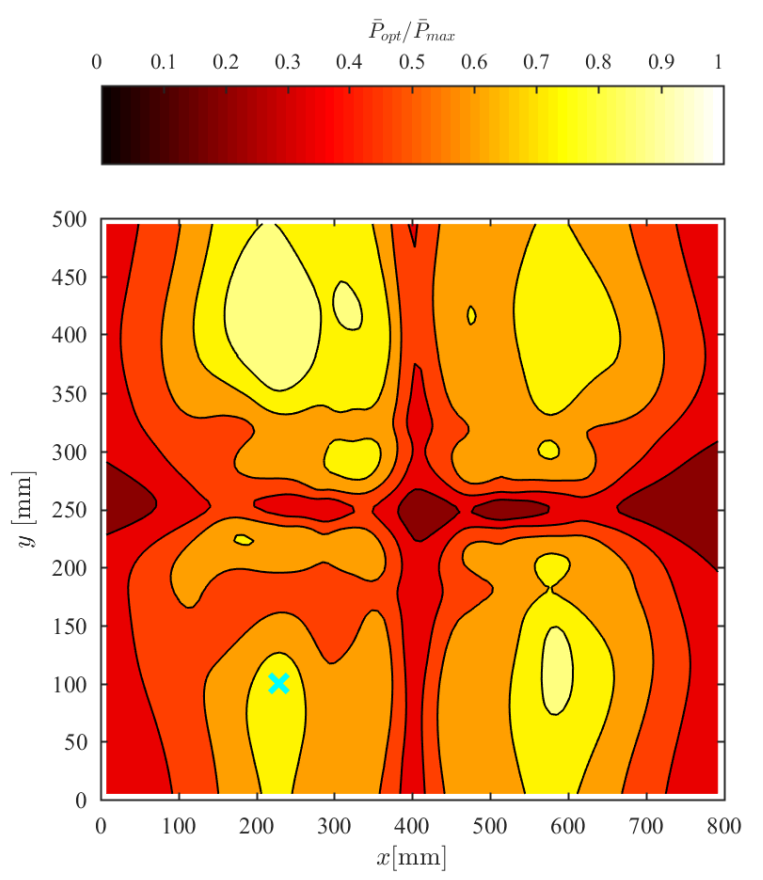

(c)

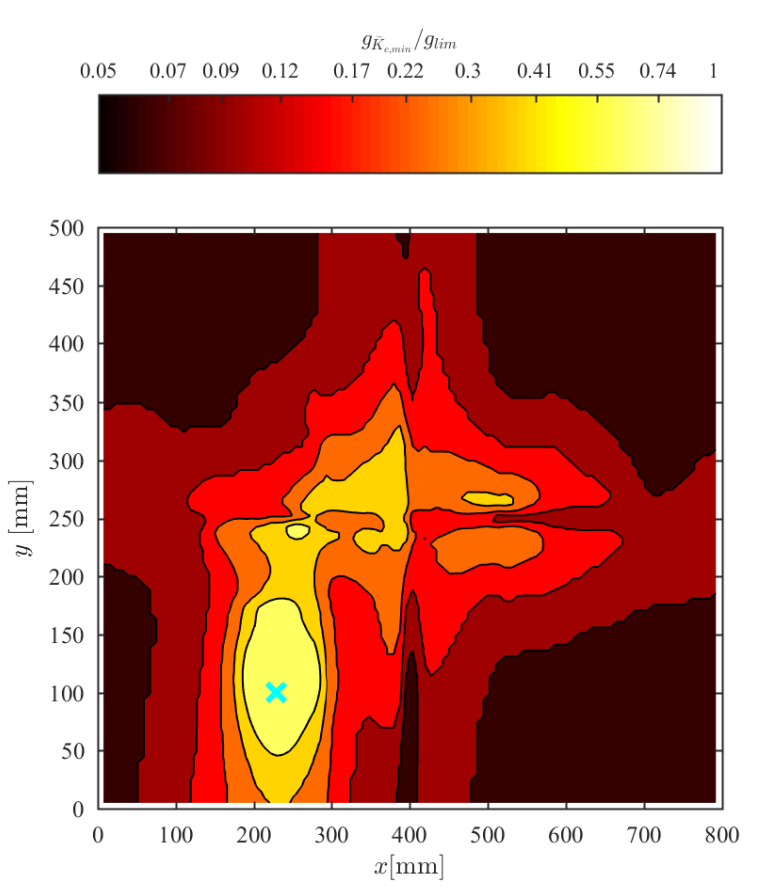

(b)

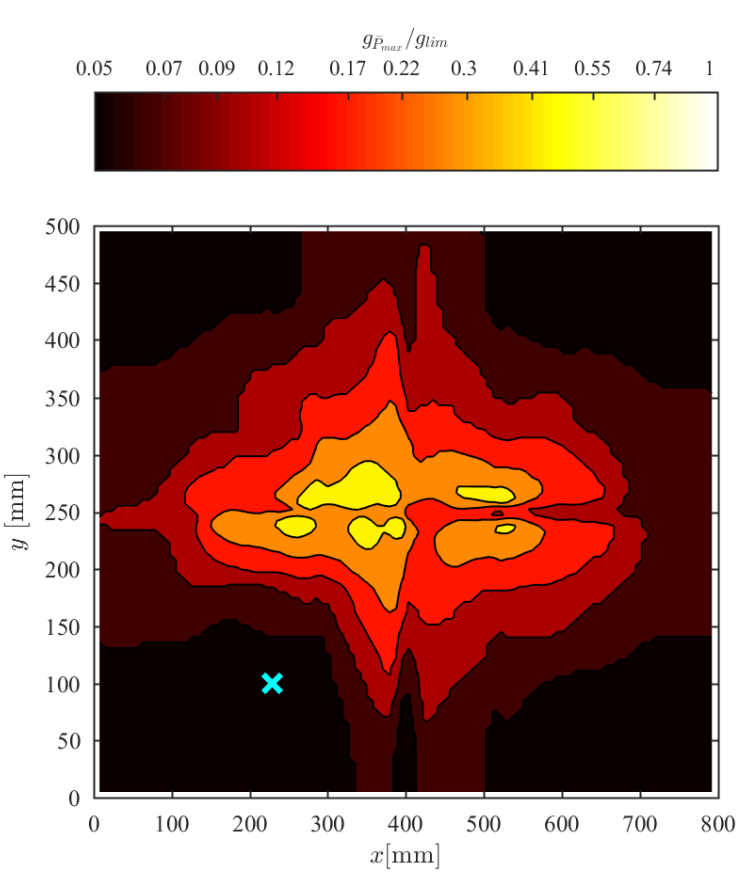

(d)

Figure A.16: Performance of a control unit at different position on a plate subjected to point excitation (cyan cross). (a) Minimum kinetic energy; (b) optimal gain minimising the kinetic energy; (c) maximum mechanical power absorbed by the control unit from the structure; (d) optimal gain maximising the mechanical power absorbed. Frequency range $0-200 \mathrm{~Hz}$. 


\section{References}

[1] L. Meirovitch, Dynamics and control of structures, John Wiley \& Sons, 1990.

[2] S. J. Elliott, Signal processing for active control, Academic press, 2000.

[3] O. N. Baumann, S. J. Elliott, An experiment comparing centralised and decentralised control using inertial actuators, ForumAcusticum 2005.

[4] W. P. Engels, O. N. Baumann, S. J. Elliott, R. Fraanje, Centralized and decentralized control of structural vibration and sound radiation, The journal of the Acoustical Society of America 119 (3) (2006) 1487-1495.

[5] N. Alujevic, P. Gardonio, K. D. Frampton, Smart double panel with decentralized active dampers for sound transmission control, AIAA journal 46 (6) (2008) 1463-1475.

[6] A. Berkhoff, J. Wesselink, Combined mimo adaptive and decentralized controllers for broadband active noise and vibration control, Mechanical Systems and Signal Processing 25 (5) (2011) 1702 - 1714. doi:https://doi.org/10.1016/j.ymssp.2010.12.012.

[7] O. N. Baumann, S. J. Elliott, The stability of decentralized multichannel velocity feedback controllers using inertial actuators, The Journal of the Acoustical Society of America 121 (1) (2007) 188-196.

[8] P. Gardonio, S. Miani, F. Blanchini, D. Casagrande, S. J. Elliott, Plate with decentralised velocity feedback loops: Power absorption and kinetic energy considerations, Journal of sound and vibration 331 (8) (2012) 1722-1741.

[9] M. Zilletti, S. J. Elliott, P. Gardonio, E. Rustighi, Experimental implementation of a self-tuning control system for decentralised velocity feedback, Journal of Sound and Vibration 331 (1) (2012) 1-14.

[10] S. Camperi, M. Ghandchi-Tehrani, S. J. Elliott, Multichannel decentralised feedback control using inertial actuators, in: Proceedings of the ISMA2016 27th International Conference on Noise and Vibration Engineering, Leuven, Belgium, 2016.

[11] M. Zilletti, S. J. Elliott, P. Gardonio, Self-tuning control systems of decentralised velocity feedback, Journal of Sound and Vibration 329 (14) (2010) 2738-2750.

[12] A. Kras, P. Gardonio, Velocity feedback control with a flywheel proof mass actuator, Journal of Sound and Vibration 402 (2017) 31-50. doi:https://doi.org/10.1016/j.jsv.2017.05.001.

[13] J. Rohlfing, S. J. Elliott, P. Gardonio, Feedback compensator for control units with proof-mass electrodynamic actuators, Journal of Sound and Vibration 331 (15) (2012) 3437 - 3450. doi:https://doi.org/10.1016/j.jsv.2012.03.010.

[14] I. Diaz, P. Reynolds, Robust saturated control of human-induced floor vibrations via a proof-mass actuator, Smart Materials and Structures 18 (12) (2009) 125024

[15] C. Collette, S. Chesne, Robust hybrid mass damper, Journal of Sound and Vibration 375 (2016) 19-27.

[16] S. J. Elliott, M. Zilletti, Scaling of electromagnetic transducers for shunt damping and energy harvesting, Journal of Sound and Vibration 333 (8) (2014) 2185-2195.

[17] S. Camperi, M. Ghandchi-Tehrani, S. J. Elliott, Investigation on the performance of a velocity feedback control unit for structural vibration control: Theory and experiments, in: Proceedings of the IMACXXXVI Conference, Orlando, Florida (USA), 2018.

[18] S. Camperi, M. Ghandchi-Tehrani, S. J. Elliott, Experimental maximisation of the power absorbed by an inertial actuator for structural vibration control, in: Proceedings of the ICSV24 24th International Congress on Sound and Vibration, London, United Kingdom, 2017.

[19] F. J. Fahy, P. Gardonio, Sound and structural vibration: radiation, transmission and response, Academic press, 2007.

[20] S. Camperi, M. Ghandchi-Tehrani, S. J. Elliott, Parametric study on the optimal tuning of an inertial actuator for vibration control of a plate: theory and experiments, in: under review for Journal of Sound and Vibration, 2018.

[21] J. M. Maciejowski, Multivariable feedback design, Addison-Wesley, Wokingham, England, 1989.

[22] S. J. Elliott, M. Zilletti, P. Gardonio, Self-tuning of local velocity feedback controllers to maximise power absorption, in: Recent Advances Structural Dynamics: Proceedings of the X International Conference, University of Southampton, 2010, p. 12pp.

[23] S. J. Elliott, M. Serrand, P. Gardonio, Feedback stability limits for active isolation systems with reactive and inertial actuators, Journal of Vibration and Acoustics 123 (2) (2001) 250-261.

[24] M. Zilletti, P. Gardonio, S. J. Elliott, Optimisation of a velocity feedback controller to minimise kinetic energy and maximise power dissipation, Journal of Sound and Vibration 333 (19) (2014) 4405-4414.

[25] W. Engels, S. Elliott, Optimal centralized and decentralized velocity feedback control on a beam, Smart Materials and Structures 17 (2) (2008) 025009. 